\title{
Radiative decays of heavy-light quarkonia through $M 1$ and $E 1$ transitions in the framework of the Bethe-Salpeter equation
}

\author{
Shashank Bhatnagar $\circledast^{1, *}$ and Eshete Gebrehana $\circledast^{2}$ \\ ${ }^{1}$ Department of Physics, University Institute of Sciences, Chandigarh University, Mohali-140413, India \\ ${ }^{2}$ Department of Physics, Addis Ababa University, P.O. Box 1176, Addis Ababa, Ethiopia
}

(Received 30 April 2020; accepted 19 October 2020; published 24 November 2020)

\begin{abstract}
In this work we study the radiative decays of heavy-light quarkonia through $M 1$ and $E 1$ transitions that involve quark-triangle diagrams with two hadron-quark vertices in the framework of $4 \times 4$ Bethe-Salpeter equation (BSE) under covariant instantaneous ansatz (CIA). We have expressed the transition amplitude $M_{f i}$ as a linear superposition of terms involving all possible combinations of ++ and -- components of Salpeter wave functions of final and initial hadron, with coefficients being related to the results of pole integrations over a complex $\sigma$ plane. We evaluate the decay widths for $M 1$ transitions $\left({ }^{3} S_{1} \rightarrow{ }^{1} S_{0}+\gamma\right)$ and $E 1$ transitions $\left({ }^{3} S_{1} \rightarrow{ }^{1} P_{0}+\gamma\right.$ and $\left.{ }^{1} P_{0} \rightarrow{ }^{3} S_{1}+\gamma\right)$. We have used algebraic forms of Salpeter wave functions obtained through analytic solutions of mass spectral equations for ground and excited states of $0^{++}, 1^{--}$, and $0^{-+}$heavy-light quarkonia in an approximate harmonic oscillator basis to calculate their decay widths. The input parameters used by us were obtained by fitting to their mass spectra. We have compared our results with experimental data and other models and found reasonable agreements.
\end{abstract}

DOI: 10.1103/PhysRevD.102.094024

\section{INTRODUCTION}

The most important goal of hadronic physics is to bridge the gap between quantum chromodynamics (QCD) Lagrangian and the observed hadronic properties. One of the challenging areas in hadronic physics presently is probing the inner structure of hadrons. There has a been a renewed interest in recent years in spectroscopy of these heavy hadrons in charm and beauty sectors, which was primarily due to experimental facilities the world over such as $B A B A R$, Belle, CLEO, DELPHI, BES, etc. [1-5], which have been providing accurate data on $c \bar{c}$ and $b \bar{b}$ hadrons with respect to their masses and decays. In the process many new states have been discovered such as $\chi_{b 0}(3 P)$, $\chi_{c 0}(2 P), X(3915), X(4260), X(4360), X(4430), X(4660)$ [5], some of which are exotic states, which cannot be readily explained through the predictions of the quark model. The radiative transitions of heavy quarkonia are of considerable experimental and theoretical interest and provide an insight into the dynamics of quarkonium. The radiative transitions between $0^{-+}$(pseudoscalar) and $1^{--}$(vector) mesons [for instance, $J / \Psi(n S) \rightarrow \eta_{c}\left(n^{\prime} S\right)+\gamma$ ], which

\footnotetext{
* Corresponding author. shashank_bhtanagar@yahoo.com

Published by the American Physical Society under the terms of the Creative Commons Attribution 4.0 International license. Further distribution of this work must maintain attribution to the author(s) and the published article's title, journal citation, and DOI. Funded by SCOAP .
}

proceeds through the emission of a photon, is characterized by $\Delta L=0$; there is change in C-parity between the initial and final hadron states, though the total C-parity is conserved. These are the magnetic dipole transitions, $M 1$. This transition mode is sensitive to relativistic effects, especially between different spatial multiplets $\left(n>n^{\prime}\right)$. The $E 1$ transitions are characterized by $|\triangle L|=1$. Thus in these transitions, there is change in parity between the initial and final hadronic states, for instance, $\Psi(2 S) \rightarrow$ $\chi_{c}(1 P)+\gamma$ or $\chi_{c}(1 P) \rightarrow J / \Psi(1 S)+\gamma$. In both $M 1$ and $E 1$ transitions, C-parity is conserved. Electric dipole transitions are much stronger than magnetic dipole transitions, and involve transitions between excited states. These transitions have been recently studied in various models, such as relativistic quark model (RQM) [6,7], effective field theory $[8,9]$, light-front quark model (LFQM) [10,11], lattice QCD $[12,13]$, and the Bethe-Salpeter equation [14-17].

In this work we focus on the radiative decays of the charmed and bottom vector mesons through the processes $V \rightarrow P \gamma, V \rightarrow S \gamma$, and $S \rightarrow V \gamma$, where $V, P, S$ refer to vector, pseudoscalar, and scalar quarkonia, and calculate the radiative decay widths of $B^{*}$ and $D^{*}$ mesons for the above mentioned processes in the framework of a $4 \times 4$ Bethe-Salpeter equation. In our recent works $[18,19]$, we studied the mass spectrum of ground and excited states of heavy-light scalar $\left(0^{++}\right)$, pseudoscalar $\left(0^{-+}\right)$, and vector $\left(1^{--}\right)$quarkonia, along with the leptonic decays of ground and excited states of $0^{-+}$and $1^{--}$quarkonia. These studies were used to fit the input parameters of our model as $C_{0}=0.69, \omega_{0}=0.22 \mathrm{GeV}, \Lambda_{\mathrm{QCD}}=0.250 \mathrm{GeV}$, and 
$A_{0}=0.01$ with input quark masses $m_{u}=0.300 \mathrm{GeV}$, $m_{s}=0.430 \mathrm{GeV}, m_{c}=1.490 \mathrm{GeV}$, and $m_{b}=4.690 \mathrm{GeV}$. In the present work on radiative decays, we use these same input parameters to calculate the single photon decay widths for the above processes.

Now, as mentioned in our previous works [17-20], we are not only interested in studying the mass spectrum of hadrons, which no doubt is an important element to study dynamics of hadrons, but also the hadronic wave functions that play an important role in the calculation of decay constants, form factors, structure functions, etc. for $Q \bar{Q}$ and $Q \bar{q}$ hadrons. These hadronic Bethe-Salpeter wave functions were calculated algebraically by us in [17-19]. The plots of these wave functions [19] show that they can not only provide information about the long distance nonperturbative physics but also act as a bridge between the long distance and short distance physics. Further, they provide us with information about the contribution of the short ranged Coulomb interactions in the mass spectral calculation of heavy-light quarkonia. These wave functions can also lead to studies on a number of processes involving $Q \bar{Q}$ and $Q \bar{q}$ states, and provide a guide for future experiments.

This paper is organized as follows: In Sec. II, we introduce the formulation of the $4 \times 4$ Bethe-Salpeter equation under the covariant instantaneous ansatz, and derive the hadron-quark vertex. In Secs. III-V, we calculate the single photon decay widths for the processes $V \rightarrow P \gamma$, $V \rightarrow S \gamma$, and $S \rightarrow V \gamma$, where $P, S$, and $V$ are the pseudoscalar, scalar, and vector heavy-light quarkonium states. In Sec. VI, we provide the numerical results and discussion.

\section{FORMULATION OF THE BSE UNDER CIA}

Our work is based on a QCD motivated BSE in ladder approximation, which is an approximate description, with an effective four-fermion interaction mediated by a gluonic propagator that serves as the kernel of a BSE in the lowest order. The precise form of our kernel is taken in analogy with potential models (PM), which includes a confining term along with a one-gluon exchange term. Such effective forms of the BS kernel in ladder BSE have recently been used in [21-25], and can predict bound states having a purely relativistic origin (as shown recently in [21]). As mentioned above, the BSE is quite general, and provides an effective description of bound quarkantiquark systems through a suitable choice of input kernel for confinement.

The Bethe-Salpeter equation that describes the quarkantiquark bound state of momenta $p_{1}$ and $p_{2}$, relative momentum $q$, and meson momentum $P$ is

$$
S_{F}^{-1}\left(p_{1}\right) \Psi(P, q) S_{F}^{-1}\left(-p_{2}\right)=i \int \frac{d^{4} q^{\prime \prime}}{(2 \pi)^{4}} K\left(q, q^{\prime \prime}\right) \Psi\left(P, q^{\prime \prime}\right),
$$

where $K\left(q, q^{\prime \prime}\right)$ is the interaction kernel and $S_{F}^{-1}\left( \pm p_{1,2}\right)=$ $\pm i \not p_{1,2}+m_{1,2}$ are the usual quark and antiquark propagators. We now make use of the covariant instantaneous ansatz (CIA) (which is a Lorentz-covariant 3D support), where $K\left(q, q^{\prime \prime}\right)=K\left(\hat{q}, \hat{q}^{\prime \prime}\right)$ on the BS kernel, and the BS kernel depends entirely on the variable. $\hat{q}_{\mu}=q_{\mu}-\frac{q \cdot P}{P^{2}} P_{\mu}$ is the component of internal momentum of the hadron that is orthogonal to the total hadron momentum, i.e. $\hat{q} \cdot P=0$, while $\sigma P_{\mu}=\frac{q \cdot P}{P^{2}} P_{\mu}$ is the component of $q$ longitudinal to $P$, where the four-dimensional volume element is $d^{4} q=d^{3} \hat{q} M d \sigma$. Now working on the right side of Eq. (1), and making use of the fact that

$$
\psi\left(\hat{q}^{\prime \prime}\right)=\frac{i}{2 \pi} \int M d \sigma^{\prime \prime} \Psi\left(P, q^{\prime \prime}\right),
$$

and the fact that the longitudinal component of $M d \sigma^{\prime \prime}$ of $q^{\prime \prime}$ does not appear in $K\left(\hat{q}, \hat{q}^{\prime \prime}\right)$, carrying out integration over $M d \sigma$ on right side of Eq. (1), we obtain

$$
\begin{aligned}
S_{F}^{-1}\left(p_{1}\right) \Psi(P, q) S_{F}^{-1}\left(-p_{2}\right) & =\int \frac{d^{3} \hat{q}^{\prime \prime}}{(2 \pi)^{3}} K\left(\hat{q}, \hat{q}^{\prime \prime}\right) \psi\left(\hat{q}^{\prime \prime}\right) \\
& =\Gamma(\hat{q}),
\end{aligned}
$$

where $\Gamma(\hat{q})$ is the hadron-quark vertex function and is directly related to the $4 \mathrm{D}$ wave function $\Psi(P, q)$, and one can express the 4D BS wave function $\Psi(P, q)$ in terms of $\Gamma(\hat{q})$ as

$$
\Psi(P, q)=S_{1}\left(p_{1}\right) \Gamma(\hat{q}) S_{2}\left(-p_{2}\right) .
$$

Further, the 4D hadron-quark vertex that enters into the definition of the 4D BS wave function in the previous equation can be identified as

$$
\Gamma(\hat{q})=\int \frac{d^{3} \hat{q}^{\prime \prime}}{(2 \pi)^{3}} K\left(\hat{q}, \hat{q}^{\prime \prime}\right) \psi\left(\hat{q}^{\prime \prime}\right) .
$$

Following a sequence of steps outlined in [17], we get four Salpeter equations which are effective 3D forms of BSE given below:

$$
\begin{aligned}
\left(M-\omega_{1}-\omega_{2}\right) \psi^{++}(\hat{q}) & =\Lambda_{1}^{+}(\hat{q}) \Gamma(\hat{q}) \Lambda_{2}^{+}(\hat{q}), \\
\left(M+\omega_{1}+\omega_{2}\right) \psi^{--}(\hat{q}) & =-\Lambda_{1}^{-}(\hat{q}) \Gamma(\hat{q}) \Lambda_{2}^{-}(\hat{q}), \\
\psi^{+-}(\hat{q}) & =0, \\
\psi^{-+}(\hat{q}) & =0 .
\end{aligned}
$$

Thus, in our framework, a crucial role is played by the component $\hat{q}_{\mu}$, which is always orthogonal to $P_{\mu}$ and satisfies the unconstrained relation $\hat{q} \cdot P=0$, regardless of whether $q \cdot P=0$ (i.e., $\sigma=0$ ) or $q \cdot P \neq 0$ (i.e., $\sigma \neq 0$ ). We further wish to point out that the effectively 3D Salpeter equations (used for mass spectral calculations), as well as 
the vertex function $\Gamma(\hat{q})$ (used for transition amplitude calculations) have a common dependence on the quantity $\hat{q}^{2}$, whose most important property is its positive definiteness $\hat{q}^{2}=q^{2}-\frac{(q . P)^{2}}{P^{2}} \geq 0$ on the hadron mass shell $\left(P^{2}=-M^{2}\right)$ throughout the entire 4D space $[14,15,24,26,27]$. Thus, $\hat{q}^{2}$ is a Lorentz-invariant variable and is a four-scalar, whose validity extends over the entire 4D space, while keeping contact with the surface $P . q=0$ (hadron rest frame), where $\hat{q}^{2}=\vec{q}^{2}$. This condition $P . q=0$ is in fact the same as instantaneous approximation.

Thus, in view of these remarkable properties of $\hat{q}_{\mu}$, which makes it an effectively 3D vector, our twin objective of (i) 3D structure of BSE as the controlling equation for spectra, and (ii) a general enough (off-shell) structure of BS vertex function $\Gamma(\hat{q})$ to facilitate applications to transition amplitudes in 4D form is largely met if the BS kernel depends on $\hat{q}_{\mu}$. Thus, the ansatz, $K\left(q, q^{\prime \prime}\right)=K\left(\hat{q}, \hat{q}^{\prime \prime}\right)$ on the BS kernel, is known as the covariant instantaneous ansatz and is a Lorentz-invariant generalization of the instantaneous approximation.

Besides the 3D Salpeter equations, the hadron-quark vertex functions $\Gamma(\hat{q})$ also have an explicit dependence on the variable, $\hat{q}^{2}$. These equations form a zero-order basis for making contact with the mass spectrum of hadronic states and calculations of various types of transition amplitudes through appropriate quark-loop diagrams.

Thus, the Lorentz-invariant nature of $\hat{q}^{2}$ increases the applicability of this framework of covariant instantaneous ansatz all the way from low energy spectra to high energy transition amplitudes. Further, in this approach, the most important aspect is the appearance of the hadron-quark vertex $\Gamma(\hat{q})$ (used for calculation of transition amplitudes) on the right side of effectively 3D Salpeter equations (used for calculation of spectra) in Eq. (6), which gives a dynamical link between low energy mass spectroscopy and high energy transition amplitudes. The dynamical links between 3D spectra and 4D transition amplitudes have been explored in detail in $[14,15,26]$ by showing the exact interconnection between the 3D and 4D BSE.

The 3D BS wave function can be expressed in terms of the projected wave functions as

$$
\psi(\hat{q})=\psi^{++}(\hat{q})+\psi^{+-}(\hat{q})+\psi^{-+}(\hat{q})+\psi^{--}(\hat{q}),
$$

where

$$
\psi^{ \pm \pm}(\hat{q})=\Lambda_{1}^{ \pm}(\hat{q}) \frac{\not p}{M} \psi(\hat{q}) \frac{\not P}{M} \Lambda_{2}^{ \pm}(\hat{q})
$$

and the projection operators

$$
\begin{aligned}
\Lambda_{j}^{ \pm}(\hat{q}) & =\frac{1}{2 \omega_{j}}\left[\frac{\not p}{M} \omega_{j} \pm J(j)\left(i m_{j}+\hat{q}\right)\right], \\
J(j) & =(-1)^{j+1}, \quad j=1,2
\end{aligned}
$$

with the relation

$$
\omega_{j}^{2}=m_{j}^{2}+\hat{q}^{2}
$$

\section{RADIATIVE DECAYS OF HEAVY-LIGHT QUARKONIA THROUGH $\boldsymbol{V} \rightarrow \boldsymbol{P} \boldsymbol{\gamma}$}

In transitions involving single photon decays, such as $V \rightarrow P+\gamma$, the process requires calculation of a triangle quark-loop diagram, which involves two hadron-quark vertices that we attempt in the $4 \times 4$ representation of a BSE. The single photon decay of vector $\left(1^{--}\right)$quarkonia is described by the direct and exchange Feynman diagrams as in Fig. 1.

To apply the framework of BSE to study radiative decays, $V \rightarrow P \gamma$, we have to remember that there are two Lorentz frames, one the rest frame of the initial meson, and the other, the rest frame of the final meson. To calculate further, we first write the relationship between the momentum variables of the initial and final meson. Here, $P$ and $q$ are the total momentum and the internal momentum of the initial hadron, while $P^{\prime}$ and $q^{\prime}$ are the corresponding variables of the final hadron. Let $k$, and $\epsilon^{\lambda^{\prime}}$ be momentum and polarization vectors of the emitted photon, while $\epsilon^{\lambda}$ is the polarization vector of the initial meson. Thus if $p_{1,2}$ and $p_{1,2}^{\prime}$ are the momenta of the two quarks in the initial and final hadron respectively, then we have the momentum relations

$$
\begin{aligned}
P=p_{1}+p_{2} ; & p_{1,2}=\hat{m}_{1,2} P \pm q, \\
P^{\prime}=p_{1}^{\prime}+p_{2}^{\prime} ; & p_{1,2}^{\prime}=\hat{m}_{1,2} P^{\prime} \pm q^{\prime}
\end{aligned}
$$

for initial and final hadrons, respectively. From the Feynman diagrams we see that conservation of momentum demands that $P=P^{\prime}+k$, while from the first diagram $p_{1}=p_{1}^{\prime}+k$ and $-p_{2}=-p_{2}^{\prime}$, where $k=P-P^{\prime}$ is the momentum of the emitted photon. Making use of the above equations, we can express the relationship between the internal momenta of the two hadrons in terms of the photon momentum $k$ as

$$
q^{\prime}=q+\left(\hat{m}_{1}-1\right) k=q-\hat{m}_{2} k
$$

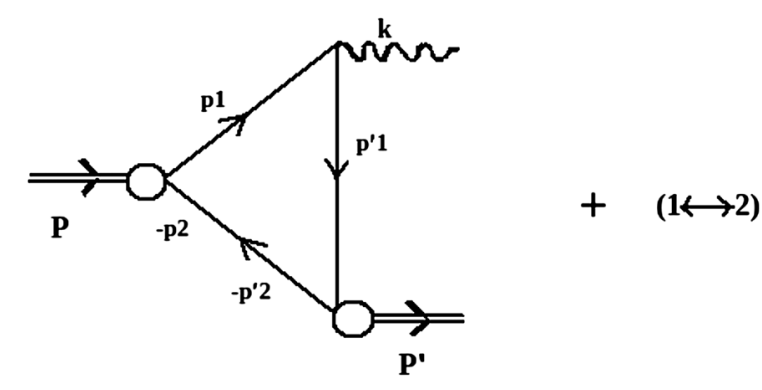

FIG. 1. Radiative decays of heavy-light quarkonia. 
where $\hat{m}_{1,2}=\frac{1}{2}\left[1 \pm \frac{\left(m_{1}^{2}-m_{2}^{2}\right)}{M^{2}}\right]$ is the Wightman-Garding definitions [26] of masses of individual quarks, which ensure that $P . q=0$ on the mass shells of either quarks, even when $m_{1} \neq m_{2}$. They act like momentum partitioning functions for the two quarks in a hadron. We had already decomposed the internal momentum $q$ of the initial hadron into two components, $q=(\hat{q}, i M \sigma)$, where $\hat{q}_{\mu}$ is the component of internal momentum transverse to $P$ such that $\hat{q} \cdot P=0$, while $\sigma$ is the longitudinal component in the direction of $P$. Similarly for the final meson, we decompose its internal momentum $q^{\prime}$ into two components $q^{\prime}=\left(\hat{q}^{\prime}, i M \sigma^{\prime}\right)$ with $\hat{q}^{\prime}=q^{\prime}-\sigma^{\prime} P$ transverse to the initial hadron momentum $P$, and $\sigma^{\prime}=\frac{q^{\prime} . P}{P^{2}}$, longitudinal to $P$. Thus, $P . \hat{q}^{\prime}=0$.

We now first try to find the relationship between the transverse components of internal momenta of the two hadrons, $\hat{q}$ and $\hat{q}^{\prime}$. For this, we resolve all momenta in Eq. (12) along the direction transverse to the momentum of the initial meson $P$. Thus we can express Eq. (12) as

$$
\begin{aligned}
\hat{q}^{\prime} & =\hat{q}+\hat{m}_{2}\left(\hat{P}^{\prime}-\hat{P}\right), \\
\hat{P} & =0, \\
\hat{P}^{\prime} & =P^{\prime}-\frac{P^{\prime} . P}{P^{2}} P,
\end{aligned}
$$

where it can be easily checked that $\hat{P} \cdot P=0$, and thus $\hat{P}^{\prime}$ is orthogonal to $P$. The above equation can be simplified as

$$
\hat{q}^{\prime}=\hat{q}+\hat{m}_{2}\left(\hat{P}^{\prime}\right) .
$$

It should be mentioned that the above relation connecting $\hat{q}$ and $\hat{q}^{\prime}$ is again consistent with the transversality of $\hat{q}^{\prime}$ with $P$, given by the definition of $\hat{q}^{\prime}$. And it can be easily checked that $\hat{q}^{\prime} . P=0$.

Now, the kinematics gets simplified in the rest frame of the initial meson, where we have $P=(\overrightarrow{0}, i M)$, while for an emitted meson, $P^{\prime}=\left(\vec{P}^{\prime}, i E^{\prime}\right)$, where $E^{\prime}=\sqrt{\vec{P}^{\prime 2}+M^{\prime 2}}$, and since the photon momentum can be decomposed as $k=(\vec{k}, i|\vec{k}|)$, where $\vec{k}=-\vec{P}^{\prime}$, since the final meson and photon would be emitted in opposite directions. Hence we get $\left|\vec{P}^{\prime}\right|=|\vec{k}|=\frac{M^{2}-M^{\prime 2}}{2 M}$. Thus the energy of the emitted meson can be expressed as $E^{\prime}=\frac{M^{2}+M^{\prime 2}}{2 M}$.

Further, the dot products of momenta of the initial and the emitted meson can be expressed as

$$
P^{\prime} . P=-M E^{\prime}=-\frac{M^{2}+M^{\prime 2}}{2} .
$$

Thus, it can be seen that $-E^{\prime}$ acts as the projection of $P^{\prime}$ along the direction of an initial hadron momentum $P$. Now, we try to find the relationship between the time components $\sigma$ and $\sigma^{\prime}$ of the two hadrons. Taking the dot product of
Eq. (12) with $P$, the momentum of the initial hadron, we obtain

$$
P . q^{\prime}=P . q-\hat{m}_{2} P . k .
$$

Making use of the above decomposition of internal momenta, we obtain the relation between the longitudinal components of internal momenta of the two hadrons as

$$
\begin{aligned}
\sigma^{\prime} & =\sigma+\alpha ; \\
\alpha & =\hat{m}_{2} \frac{M^{\prime 2}-M^{2}}{2 M^{2}},
\end{aligned}
$$

which is again a consequence of the transversality of $\hat{q}^{\prime}$ with initial hadron momentum $P$. Thus, up to Eq. (17), the kinematics is the same for all the three processes $(V \rightarrow P \gamma$, $V \rightarrow S \gamma$, and $S \rightarrow V \gamma$ ) studied in this work.

It is to be noted that 4D BS wave functions of a vector meson involved in the process (in variable $\hat{q}$ ) is exactly the same as Eq. (4), with $\Psi(P, q) \Rightarrow \Psi_{V}(P, q)$ and $\Gamma(\hat{q}) \Rightarrow \Gamma_{V}(\hat{q})$. However, for transition amplitude calculation, we are choosing to do the calculation in the rest frame of the initial meson. Thus, we write

$$
\Psi_{P}\left(P^{\prime}, q^{\prime}\right)=S_{F}\left(p_{1}^{\prime}\right) \Gamma_{P}\left(\hat{q}^{\prime}\right) S_{F}\left(-p_{2}^{\prime}\right)
$$

with $\hat{q}^{\prime}$ defined earlier as $\hat{q}^{\prime}=q^{\prime}-\frac{q^{\prime} . P}{P^{2}} P$, that is transverse to initial hadron momentum $P$, where the hadron-quark vertex function $\Gamma_{V}\left(\hat{q}^{\prime}\right)$ for the final meson is

$$
\Gamma\left(\hat{q}^{\prime}\right)=\int \frac{d^{3} \hat{q}^{\prime \prime \prime}}{(2 \pi)^{3}} K\left(\hat{q}^{\prime}, \hat{q}^{\prime \prime \prime}\right) \psi\left(\hat{q}^{\prime \prime \prime}\right) .
$$

Similarly for final meson, the expression for the 3D BS wave function $\psi\left(\hat{q}^{\prime}\right)$ is expressed in terms of the projection operators as

$\psi\left(\hat{q}^{\prime}\right)=\psi^{++}\left(\hat{q}^{\prime}\right)+\psi^{+-}\left(\hat{q}^{\prime}\right)+\psi^{-+}\left(\hat{q}^{\prime}\right)+\psi^{--}\left(\hat{q}^{\prime}\right)$

where

$$
\begin{aligned}
\psi^{ \pm \pm}\left(\hat{q}^{\prime}\right) & =\Lambda_{1}^{ \pm}\left(\hat{q}^{\prime}\right) \frac{\not p}{M} \psi\left(\hat{q}^{\prime}\right) \frac{\not P}{M} \Lambda_{2}^{ \pm}\left(\hat{q}^{\prime}\right), \\
\Lambda_{j}^{ \pm}\left(\hat{q}^{\prime}\right) & =\frac{1}{2 \omega_{j}^{\prime}}\left[\frac{\not P}{M} \omega_{j}^{\prime} \pm J(j)\left(i m_{j}+\hat{q}^{\prime}\right)\right],
\end{aligned}
$$

with the relation $\omega_{1,2}^{\prime 2}=m_{1,2}^{2}+\hat{q}^{\prime 2}$.

The EM transition amplitude of the process is

$$
\begin{aligned}
M_{f i}= & -i \int \frac{d^{4} q}{(2 \pi)^{4}} \operatorname{Tr}\left[e_{q} \bar{\Psi}_{P}\left(P^{\prime}, q^{\prime}\right) \phi^{\lambda^{\prime}} \Psi_{V}(P, q)\right. \\
& \left.\times S_{F}^{-1}\left(-p_{2}\right)+e_{\bar{Q}} \bar{\Psi}_{P}\left(P^{\prime}, q^{\prime}\right) S_{F}^{-1}\left(p_{1}\right) \Psi_{V}(P, q) \phi^{\lambda^{\prime}}\right] .
\end{aligned}
$$


Here, the first term corresponds to the first diagram, where the photon is emitted from the quark, while the second term corresponds to the second diagram where the photon is emitted from the antiquark.

In the above expression, $\Psi_{P}$ and $\Psi_{V}$ are the 4D BS wave functions of pseudoscalar and vector quarkonia involved in the process, and are expressed above, while $e_{q}$ and $e_{Q}$ are the electric charge of quark and antiquark, respectively, and $\epsilon_{\mu}^{\lambda^{\prime}}$ is the polarization vector of the emitted photon.

Using the fact that the contribution of the second term is the same as that of the first term (except that $e_{q} \neq e_{\bar{Q}}$ ), we rewrite the above equation in terms of the electronic charge $e$ as
$M_{f i}=-i e \int \frac{d^{4} q}{(2 \pi)^{4}} \operatorname{Tr}\left[\bar{\Psi}_{P}\left(P^{\prime}, q^{\prime}\right) \phi^{\prime \prime} \Psi_{V}(P, q) S_{F}^{-1}\left(-p_{2}\right)\right]$.

Now, we reduce the above equation to the effective 3D form by integrating over the longitudinal component $M d \sigma$. This can be expressed as

$$
\begin{aligned}
M_{f i}= & -i e \int \frac{d^{3} \hat{q}}{(2 \pi)^{3}} \int \frac{i M d \sigma}{(2 \pi)} \operatorname{Tr}\left[\bar{\Gamma}_{P}\left(\hat{q}^{\prime}\right) S_{F}\left(p_{1}^{\prime}\right) \phi^{\prime \prime} S_{F}\left(p_{1}\right)\right. \\
& \left.\times \Gamma_{V}(\hat{q}) S_{F}\left(-p_{2}\right)\right]
\end{aligned}
$$

To calculate $M_{f i}$, we express the propagators $S_{F}$ as

$$
\begin{aligned}
S_{F}\left(p_{1}\right) & =\frac{\Lambda_{1}^{+}(\hat{q})}{M \sigma+\hat{m}_{1} M-\omega_{1}+i \epsilon}+\frac{\Lambda_{1}^{-}(\hat{q})}{M \sigma+\hat{m}_{1} M+\omega_{1}-i \epsilon}, \\
S_{F}\left(-p_{2}\right) & =\frac{-\Lambda_{2}^{+}(\hat{q})}{-M \sigma+\hat{m}_{2} M-\omega_{2}+i \epsilon}+\frac{-\Lambda_{2}^{-}(\hat{q})}{-M \sigma+\hat{m}_{2} M+\omega_{2}-i \epsilon}, \\
S_{F}\left(p_{1}^{\prime}\right) & =\frac{\Lambda_{1}^{+}\left(\hat{q}^{\prime}\right)}{M \sigma^{\prime}+\hat{m}_{1}\left(-E^{\prime}\right)-\omega_{1}^{\prime}+i \epsilon}+\frac{\Lambda_{1}^{-}(\hat{q})^{\prime}}{M \sigma^{\prime}+\hat{m}_{1}\left(-E^{\prime}\right)+\omega_{1}^{\prime}-i \epsilon} .
\end{aligned}
$$

It should be noted that the expression for $M_{f i}$ in Eq. (24) is written down in the frame of the initial hadron. We wish to mention that, from the very beginning, we have used the relationship that connects the internal momentum $q^{\prime}=$ $\left(\hat{q}^{\prime}, i M \sigma^{\prime}\right)$ of the final hadron with the internal momentum $q=(\hat{q}, i M \sigma)$ of the initial hadron, given by Eq. (12). As mentioned earlier, the space component $\hat{q}^{\prime}$ of $q^{\prime}$ is transverse to the total momentum $P$ of the initial hadron, while the time component $M \sigma^{\prime}$ is longitudinal to $P$. A consequence of Eq. (12) is that $\hat{q}^{\prime}$ and $\hat{q}$ are related to each other by Eq. (14), while the time component $\sigma^{\prime}$ is related to $\sigma$ through Eq. (17). Thus, these relations, Eqs. (12), (14), and
(17), are made use of to express $M_{f i}$ as a single integral over $d^{4} q=d^{3} \hat{q} M d \sigma$, while transforming $\sigma^{\prime}$ that enters through the propagator $S_{F}\left(p_{1}^{\prime}\right)$ in terms of $\sigma$ through Eq. (17). We then carry out the contour integral over $M d \sigma$ over the poles of the propagators in Eqs. (24) and (26) to finally obtain Eq. (31).

We now put the propagators into Eq. (24), and multiplying this equation from the left by the relation $\frac{\not p}{M} \frac{\not p}{M}=$ $-1=\frac{\not p}{M}\left(\Lambda_{2}^{+}\left(\hat{q}^{\prime}\right)+\Lambda_{2}^{-}\left(\hat{q}^{\prime}\right)\right)$ [24], and making use of Eq. (17) to transform $\sigma^{\prime}$ to $\sigma$ through relation $\sigma^{\prime}=\sigma+\alpha$, the transition amplitude can be expressed as

$$
\begin{aligned}
M_{f i} & =-i e \int \frac{d^{3} \hat{q}}{(2 \pi)^{3}}\left[\Omega_{1}+\Omega_{2}+\Omega_{3}+\Omega_{4}\right], \\
\Omega_{1} & =\int \frac{d \sigma}{(2 \pi)} \frac{i}{M^{3}} \operatorname{Tr}\left[\frac{-\not P \Lambda_{2}^{+}\left(\hat{q}^{\prime}\right) \bar{\Gamma}_{P}\left(\hat{q}^{\prime}\right) \Lambda_{1}^{+}\left(\hat{q}^{\prime}\right) \phi^{\prime \prime} \Lambda_{1}^{+}(\hat{q}) \Gamma_{V}(\hat{q}) \Lambda_{2}^{+}(\hat{q})}{\left[\sigma-\left(-\alpha+\hat{m}_{1} \frac{E^{\prime}}{M}+\frac{\omega_{1}^{\prime}}{M}\right)\right]\left[\sigma-\left(-\hat{m}_{1}+\frac{\omega_{1}}{M}\right)\right]\left[\sigma-\left(\hat{m}_{2}-\frac{\omega_{2}}{M}\right)\right]}\right], \\
\Omega_{2} & =\int \frac{d \sigma}{(2 \pi)} \frac{i}{M^{3}} \operatorname{Tr}\left[\frac{-\not P \Lambda_{2}^{+}\left(\hat{q}^{\prime}\right) \bar{\Gamma}_{P}\left(\hat{q}^{\prime}\right) \Lambda_{1}^{+}\left(\hat{q}^{\prime}\right) \phi^{\prime \prime} \Lambda_{1}^{-}(\hat{q}) \Gamma_{V}(\hat{q}) \Lambda_{2}^{-}(\hat{q})}{\left[\sigma-\left(-\alpha+\hat{m}_{1} \frac{E^{\prime}}{M}+\frac{\omega_{1}^{\prime}}{M}\right)\right]\left[\sigma-\left(-\hat{m}_{1}-\frac{\omega_{1}}{M}\right)\right]\left[\sigma-\left(\hat{m}_{2}+\frac{\omega_{2}}{M}\right)\right]}\right], \\
\Omega_{3} & =\int \frac{d \sigma}{(2 \pi)} \frac{i}{M^{3}} \operatorname{Tr}\left[\frac{-\not P \Lambda_{2}^{-}\left(\hat{q}^{\prime}\right) \bar{\Gamma}_{P}\left(\hat{q}^{\prime}\right) \Lambda_{1}^{-}\left(\hat{q}^{\prime}\right) \phi^{\prime \prime} \Lambda_{1}^{+}(\hat{q}) \Gamma_{V}(\hat{q}) \Lambda_{2}^{+}(\hat{q})}{\left[\sigma-\left(-\alpha+\hat{m}_{1} \frac{E^{\prime}}{M}-\frac{\omega_{1}^{\prime}}{M}\right)\right]\left[\sigma-\left(-\hat{m}_{1}+\frac{\omega_{1}}{M}\right)\right]\left[\sigma-\left(\hat{m}_{2}-\frac{\omega_{2}}{M}\right)\right]}\right], \\
\Omega_{4} & =\int \frac{d \sigma}{(2 \pi)} \frac{i}{M^{3}} \operatorname{Tr}\left[\frac{-\not P \Lambda_{2}^{+}\left(\hat{q}^{\prime}\right) \bar{\Gamma}_{P}\left(\hat{q}^{\prime}\right) \Lambda_{1}^{-}\left(\hat{q}^{\prime}\right) \phi^{\prime \prime} \Lambda_{1}^{-}(\hat{q}) \Gamma_{V}(\hat{q}) \Lambda_{2}^{-}(\hat{q})}{\left[\sigma-\left(-\alpha+\hat{m}_{1} \frac{E^{\prime}}{M}-\frac{\omega_{1}^{\prime}}{M}\right)\right]\left[\sigma-\left(-\hat{m}_{1}-\frac{\omega_{1}}{M}\right)\right]\left[\sigma-\left(\hat{m}_{2}+\frac{\omega_{2}}{M}\right)\right]}\right],
\end{aligned}
$$


where the rest of the terms are anticipated to be zero on account of 3D Salpeter equations. The contour integrations over $M d \sigma$ are performed over each of the four terms taking into account the pole positions in the complex $\sigma$ plane:

$$
\begin{aligned}
& \sigma_{3}^{ \pm}=-\alpha+\hat{m}_{1} \frac{E^{\prime}}{M} \mp \frac{\omega_{1}^{\prime}}{M} \pm i \epsilon, \\
& \sigma_{1}^{ \pm}=-\hat{m}_{1} \mp \frac{\omega_{1}}{M} \pm i \epsilon, \\
& \sigma_{2}^{ \pm}=\hat{m}_{2} \mp \frac{\omega_{2}}{M} \pm i \epsilon .
\end{aligned}
$$

In Eq. (26), the contour integral over each of the four terms can be performed by closing the contour either above or below the real axis in the complex $\sigma$ plane with pole positions displayed in Fig. 2. We demonstrate, for instance, the calculation of $\Omega_{1}$ in which the poles $\sigma_{3}^{-}$and $\sigma_{1}^{-}$are

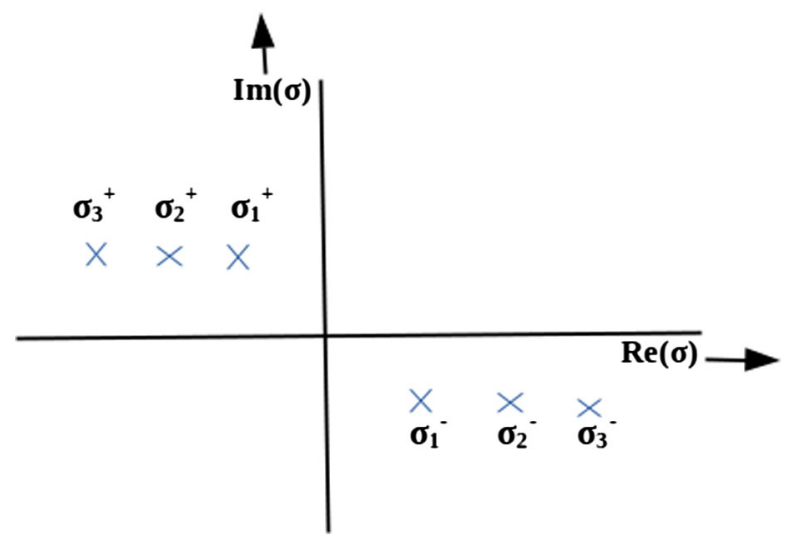

FIG. 2. Pole positions in the complex $\sigma$ plane.

below the real axis, while $\sigma_{2}^{+}$is above the real axis in the complex $\sigma$ plane. The result of $\Omega_{1}$ integration is $I_{1}$ given below:

$$
\begin{aligned}
\Omega_{1} & =\operatorname{Tr}\left[-\not P\left[\Lambda_{2}^{+}\left(\hat{q}^{\prime}\right) \bar{\Gamma}_{P}\left(\hat{q}^{\prime}\right) \Lambda_{1}^{+}\left(\hat{q}^{\prime}\right)\right] \phi^{\prime \prime}\left[\Lambda_{1}^{+}(\hat{q}) \Gamma_{V}(\hat{q}) \Lambda_{2}^{+}(\hat{q})\right] I_{1}\right], \\
I_{1} & =\int \frac{i}{2 \pi M^{3}} \frac{d \sigma}{\left[\sigma-\left(-\alpha+\hat{m}_{1} \frac{E^{\prime}}{M}+\frac{\omega_{1}^{\prime}}{M}\right)\right]\left[\sigma-\left(-\hat{m}_{1}+\frac{\omega_{1}}{M}\right)\right]\left[\sigma-\left(\hat{m}_{2}-\frac{\omega_{2}}{M}\right)\right]} \\
& =-\frac{1}{M^{2}} \frac{1}{\left[M-\omega_{1}-\omega_{2}\right]\left[\alpha-\hat{m}_{1} \frac{E^{\prime}}{M}+\hat{m}_{2}-\frac{1}{M}\left(\omega_{1}^{\prime}+\omega_{2}\right)\right]} .
\end{aligned}
$$

We now make use of the Salpeter equations in variable $\hat{q}$ in Eq. (6), and the Salpeter equations in variable $\hat{q}^{\prime}$ given below. It is to be noted that the Salpeter equations in $\hat{q}^{\prime}$ involve $-E^{\prime}=\frac{P . P^{\prime}}{M}$, which is the projection of $P^{\prime}$ along the direction of initial momentum $P$ and are given as [24]

$$
\begin{aligned}
\left(-E^{\prime}-\omega_{1}^{\prime}-\omega_{2}^{\prime}\right) \psi^{++}\left(\hat{q}^{\prime}\right) & =\Lambda_{1}^{+}\left(\hat{q}^{\prime}\right) \Gamma\left(\hat{q}^{\prime}\right) \Lambda_{2}^{+}\left(\hat{q}^{\prime}\right), \\
\left(-E^{\prime}+\omega_{1}^{\prime}+\omega_{2}\right) \psi^{--}\left(\hat{q}^{\prime}\right) & =-\Lambda_{1}^{-}\left(\hat{q}^{\prime}\right) \Gamma\left(\hat{q}^{\prime}\right) \Lambda_{2}^{-}\left(\hat{q}^{\prime}\right), \\
\psi^{+-}\left(\hat{q}^{\prime}\right) & =0, \\
\psi^{-+}\left(\hat{q}^{\prime}\right) & =0 .
\end{aligned}
$$

It can then be verified that $\Omega_{1}$ can be expressed as

$$
\begin{aligned}
\Omega_{1} & =\frac{1}{M^{2}} \alpha_{1} \not p \bar{\psi}_{P}^{++}\left(\hat{q}^{\prime}\right) \phi^{\prime \prime} \psi_{V}^{++}(\hat{q}) ; \\
\alpha_{1} & =\frac{\left[-E^{\prime}-\omega_{1}^{\prime}-\omega_{2}^{\prime}\right]}{\left[\alpha-\hat{m}_{1} \frac{E^{\prime}}{M}+\hat{m}_{2}-\frac{1}{M}\left(\omega_{1}^{\prime}+\omega_{2}\right)\right]} .
\end{aligned}
$$

Similarly, it can be verified that the results of each of these four integrals $\Omega_{1}, \ldots, \Omega_{4}$ whether we close the contour above or below the real $\sigma$ axis comes out to be the same, thereby validating the correctness of the formalism employed. These results of integrals over $d \sigma$ in $\Omega_{1}, \ldots, \Omega_{4}$ are given as $\alpha_{1}, \ldots, \alpha_{4}$ in Eq. (32).
This leads to the expression for the effective 3D form of the transition amplitude $M_{f i}$ under a covariant instantaneous ansatz as

$$
\begin{aligned}
M_{f i}= & -i e \int \frac{d^{3} \hat{q}}{(2 \pi)^{3}} \frac{1}{M^{2}} \operatorname{Tr}\left[\alpha_{1} \not P \bar{\psi}_{P}^{++}\left(\hat{q}^{\prime}\right) \phi^{\prime \prime} \psi_{V}^{++}(\hat{q})\right. \\
& +\alpha_{2} \not P \bar{\psi}_{P}^{++}\left(\hat{q}^{\prime}\right) \phi^{\prime \prime} \psi_{V}^{--}(\hat{q})+\alpha_{3} \not P \bar{\psi}_{P}^{--}\left(\hat{q}^{\prime}\right) \phi^{\prime \prime} \psi_{V}^{++}(\hat{q}) \\
& \left.+\alpha_{4} \not P \bar{\psi}_{P}^{--}\left(\hat{q}^{\prime}\right) \phi^{\prime \prime} \psi_{V}^{--}(\hat{q})\right] .
\end{aligned}
$$

where:

$$
\begin{aligned}
& \alpha_{1}=\frac{\left[-E^{\prime}-\omega_{1}^{\prime}-\omega_{2}^{\prime}\right]}{\left[\alpha-\hat{m}_{1} \frac{E^{\prime}}{M}+\hat{m}_{2}-\frac{1}{M}\left(\omega_{1}^{\prime}+\omega_{2}\right)\right]}, \\
& \alpha_{2}=\frac{-\left[-E^{\prime}-\omega_{1}^{\prime}-\omega_{2}^{\prime}\right]}{\left[\alpha-\hat{m}_{1}\left(\frac{E^{\prime}}{M}-1\right)-\frac{1}{M}\left(\omega_{1}+\omega_{1}^{\prime}\right)\right]}, \\
& \alpha_{3}=\frac{\left[-E^{\prime}+\omega_{1}^{\prime}+\omega_{2}^{\prime}\right]}{\left[\alpha-\hat{m}_{1}\left(\frac{E^{\prime}}{M}-1\right)+\frac{1}{M}\left(\omega_{1}+\omega_{1}^{\prime}\right)\right]}, \\
& \alpha_{4}=\frac{-\left[-E^{\prime}+\omega_{1}^{\prime}+\omega_{2}^{\prime}\right]}{\left[\alpha-\hat{m}_{1} \frac{E^{\prime}}{M}+\hat{m}_{2}+\frac{1}{M}\left(\omega_{1}^{\prime}+\omega_{2}\right)\right]},
\end{aligned}
$$

with the projected wave functions $\psi^{ \pm \pm}$being taken from the 3D Salpeter equations [19] derived earlier, which for 
the initial meson in the internal variable $\hat{q}$ are given in Eq. (6).

[It is to be noted that the factors $\left(M \pm \omega_{1} \pm \omega_{2}\right)$ that were also present in the numerators of $\alpha$ 's in Eq. (32) as a result of the first two Salpeter equations in variable $\hat{q}$ in Eq. (6) get cancelled from the corresponding factors (in denominator) resulting from contour integrals over $d \sigma$, while the numerators of $\alpha_{1}, \ldots, \alpha_{4}$ come from the Salpeter equations in variable $\hat{q}^{\prime}$ in Eq. (29).]

Thus, we have given a generalized method for handling quark-triangle diagrams with two hadron-quark vertices in the framework of a $4 \times 4$ BSE under a covariant instantaneous ansatz by expressing the transition amplitude $M_{f i}$ [Eq. (31)-(32)] as a linear superposition of terms involving all possible combinations of ++ and -- components of Salpeter wave functions of final and initial hadrons through not only the ++++ and ---- terms but also terms like ++-- and --++ , with each of the four terms being associated with a coefficient, $\alpha_{i}(i=1, \ldots, 4)$, which is the result of pole integration in the complex $\sigma$ plane, with pole positions in Eq. (27) (shown in Fig. 2). This superposition of all possible terms in Eqs. (31)-(32) should be a feature of relativistic frameworks.

Now, to calculate the process, we need the 4D BS wave functions for vector and pseudoscalar mesons. We again start with the general 4D decomposition of BS wave functions [28]. Using 3D decomposition under a covariant instantaneous ansatz, the wave function of vector mesons of dimensionality $M$ can be written as $[17,18]$

$$
\begin{aligned}
\psi^{V}(\hat{q})= & i M \phi \chi_{1}(\hat{q})+\phi \not P \chi_{2}(\hat{q})+[\dot{\phi} \hat{\phi}-\hat{q} . \epsilon] \chi_{3}(\hat{q}) \\
& -i[\not \supset \phi \hat{q}+\hat{q} . \epsilon P] \frac{1}{M} \chi_{4}(\hat{q})+(\hat{q} . \epsilon) \chi_{5}(\hat{q}) \\
& -i \hat{q} . \epsilon \frac{\not P}{M} \chi_{6}(\hat{q}),
\end{aligned}
$$

where $\epsilon^{\lambda}$ is the vector meson polarization vector. Similarly for a pseudoscalar meson, the 3D wave function with dimensionality $M$ can be written as

$$
\psi^{P}(\hat{q})=N_{P}\left[M \phi_{1}(\hat{q})-i \not P \phi_{2}(\hat{q})+i \hat{\phi} \phi_{3}(\hat{q})+\frac{\not p}{M} \phi_{4}(\hat{q})\right] \gamma_{5} .
$$

We wish to mention that in our previous works [17-19] we had calculated the mass spectrum of vector, pseudoscalar, and scalar mesons by using a full Dirac structure of wave functions in Eqs. (33), (34), and (48), respectively, into the 3D Salpeter equations in Eq. (6) and obtained the coupled Salpeter equations in the amplitudes of various Dirac structures, which were then decoupled using heavyquark approximation. Also, mass spectral equations were obtained in an approximate harmonic oscillator basis, which were used not only to calculate the mass spectrum but also to analytically derive the algebraic forms of wave functions [17,18] in Eq. (37) for both pseudoscalar and vector quarkonia, and in Eq. (50) for scalar mesons [19] in an approximate harmonic basis. These algebraic wave functions were used to calculate various transitions [17-19] by fixing the parameters of the model to the mass spectrum. Also the plots of these wave functions were studied in detail in these works. It is these very analytic forms of wave functions in Eqs. (37) and (50) that we are now using to calculate the $M 1$ and $E 1$ transitions in this work.

However, we further wish to mention that in some of the recent works [29], it was noticed that among all Dirac covariants in a structure of hadronic BS wave function, some covariants contribute much more than others in calculation of hadronic observables. This led us to develop a naive power counting rule in $[26,30,31]$ by which one could classify various Dirac structures as leading and subleading. Thus, in our framework [26,30,31], we had shown that in case of pseudoscalar mesons, the Dirac structures associated with amplitudes $\phi_{1}$ and $\phi_{2}$ are leading, while the structures associated with $\phi_{3}$ and $\phi_{4}$ are subleading. In various calculations [26,30-33] it was shown that the Dirac structure associated with $\phi_{1}$ (i.e., $\gamma_{5}$ ) is most dominant, for not only ground state pseudoscalar mesons but also their excited states, and this is more true for heavy mesons.

A similar behavior was observed in the case of vector mesons [26,30], where structures associated with $\chi_{1}$ and $\chi_{2}$ are leading, while those associated with $\chi_{3}, \ldots, \chi_{6}$ are subleading, and among the leading Dirac structures, the structure associated with $\chi_{1}$ (i.e., $i \gamma . \epsilon$ ) is the most dominant. These dominant Dirac structures contribute nearly $80 \%-90 \%$ to the calculation of any meson observable, and their contribution [26,29-31] increases with an increase in meson mass. Further, such dominant Dirac structures, such as $\Gamma=\gamma_{5}\left(\right.$ for $\left.0^{-+}\right), \gamma_{\mu}$ (for $1^{--}$), and 1 (for $0^{++}$), have also been used recently in lattice calculations [34] of radiative decays of charmonium.

Now, it does appear that when one uses only the dominant Dirac structures, we do not need all four Salpeter equations, and we could have just used the first of the Salpeter equations in Eq. (6). But if we do this, we cannot work out the spectral problem analytically in an approximate harmonic oscillator basis, which has the advantage of exhibiting a greater transparency by providing an explicit dependence of the mass spectrum on the principle quantum number $N$, which gives a much deeper insight, and we would have to resort to numerical analysis at an early stage for calculating the mass spectrum. Further, we would also not be able to obtain the algebraic forms of wave functions in Eqs. (37) and (50) (that arise as solutions of the mass spectral equation in an approximate harmonic basis) that are in turn used to calculate various transition amplitudes.

Thus to simplify the algebra, we take the most dominant Dirac structures, while keeping the radial wave functions 
the same as those obtained through solutions of the full spectral problem. This might lead to a little loss of numerical accuracy, but the major gain is in retaining the explicit dependence of mass spectrum on the principle quantum number $N$ and algebraic forms of wave functions that are used for all transition amplitude calculations.

Thus, the 3D Bethe-Salpeter wave functions of heavylight pseudoscalar and vector quarkonia are taken as

$$
\begin{aligned}
& \psi_{P}(\hat{q})=N_{P}\left(M \gamma_{5}\right) \phi_{P}(\hat{q}), \\
& \psi_{V}(\hat{q})=N_{V}(i M \phi) \phi_{V}(\hat{q}) .
\end{aligned}
$$

The Bethe-Salpeter normalizers obtained through the current conservation condition are

$$
\begin{aligned}
& N_{P}^{-2}=4 \hat{m}_{1} \hat{m}_{2} M^{\prime 2} \frac{1}{m_{1}} \int \frac{d^{3} \hat{q}}{(2 \pi)^{3}} \phi_{P}^{2}(\hat{q}), \\
& N_{V}^{-2}=4 \hat{m}_{1} \hat{m}_{2} M^{2} \frac{1}{m_{1}} \int \frac{d^{3} \hat{q}}{(2 \pi)^{3}} \phi_{V}^{2}(\hat{q}) .
\end{aligned}
$$

The 3D wave functions of ground and excited states of pseudoscalar $0^{-+}$and vector $1^{--}$quarkonia are [19]

$$
\begin{aligned}
\phi_{P, V}(1 S, \hat{q}) & =\frac{1}{\pi^{3 / 4}} \frac{1}{\beta_{P, V}^{3 / 2}} e^{-\frac{\hat{q}^{2}}{2 \hat{\beta}_{P, V}}}, \\
\phi_{P, V}(2 S, \hat{q}) & =\sqrt{\frac{3}{2}} \frac{1}{\pi^{3 / 4}} \frac{1}{\beta_{P, V}^{3 / 2}}\left(1-\frac{2 \hat{q}^{2}}{3 \beta_{P, V}^{2}}\right) e^{-\frac{\hat{q}^{2}}{2 \beta_{P, V}^{2}}}, \\
\phi_{V}(1 D, \hat{q}) & =\sqrt{\frac{4}{15}} \frac{1}{\pi^{3 / 4}} \frac{1}{\beta_{V}^{7 / 2}} \hat{q}^{2} e^{-\frac{\hat{q}^{2}}{2 \beta_{V}^{2}}}, \\
\phi_{P, V}(3 S, \hat{q}) & =\sqrt{\frac{15}{8}} \frac{1}{\pi^{3 / 4}} \frac{1}{\beta_{P, V}^{3 / 2}}\left(1-\frac{4 \hat{q}^{2}}{3 \beta_{P, V}^{2}}+\frac{4 \hat{q}^{4}}{15 \beta_{P, V}^{4}}\right) e^{-\frac{\hat{q}^{2}}{2 \beta_{P, V}^{2}}},
\end{aligned}
$$

where $\beta_{P, V}$ [19] are the inverse range parameters.

We have made use of the 3D Salpeter equations in Eq. (6) that depend on the variable $\hat{q}^{2}$, that (as explained in Sec. II) is Lorentz invariant and is a four-scalar, whose validity extends over the entire 4D space, while also keeping contact with the surface $P . q=0$ (hadron rest frame). Now, our mass spectrum, and the 3D wave functions $\phi(\hat{q})$ in Eqs. (37) and (50) (please see [17]) were calculated from Salpeter equations in Eq. (6) in the rest frame of the hadron.

The ++ and -- components of the BS wave function for a pseudoscalar meson are $[17,24]$

$$
\psi_{P}^{ \pm \pm}\left(\hat{q}^{\prime}\right)=\Lambda_{1}^{ \pm}\left(\hat{q}^{\prime}\right) \frac{\not P}{M} \psi_{P}\left(\hat{q}^{\prime}\right) \frac{\not P}{M} \Lambda_{2}^{ \pm}\left(\hat{q}^{\prime}\right) .
$$

Substituting the 3D BS wave function of a pseudoscalar meson, the ++ and -- components of the $3 \mathrm{D}$ BS wave function of a pseudoscalar meson can be obtained using Eq. (38) as given in Eq. (A1) of Appendix A. 1. The corresponding adjoint wave functions are given in Eq. (A2).

Whereas the positive and negative energy components of the vector meson wave function are

$$
\psi_{V}^{ \pm \pm}(\hat{q})=\Lambda_{1}^{ \pm}(\hat{q}) \frac{\not p}{M} \psi_{V}(\hat{q}) \frac{\not p}{M} \Lambda_{2}^{ \pm}(\hat{q})
$$

Following the same steps as in Eq. (A3), we obtain the ++ and -- components of the 3D BS wave function of a vector meson through Eq. (39). These components of a vector meson wave function are given in Eq. (A3), and their corresponding adjoint wave functions are given in Eq. (A4).

We now calculate the individual terms, $\not P \bar{\psi}_{P}^{++}\left(\hat{q}^{\prime}\right) \phi^{\prime \prime}$ $\Psi_{V}^{++}(\hat{q}), \quad \not p \bar{\psi}_{P}^{++}\left(\hat{q}^{\prime}\right) \phi^{\prime \prime} \Psi_{V}^{--}(\hat{q}), \quad \not p \bar{\psi}_{P}^{--}\left(\hat{q}^{\prime}\right) \phi^{\prime} \Psi_{V}^{++}(\hat{q})$, and $\not p \bar{\psi}_{P}^{--}\left(\hat{q}^{\prime}\right) \phi^{\prime} \Psi_{V}^{--}(\hat{q})$ in the transition amplitude $M_{f i}$. These terms are given in Eqs. (A5)-(A8).

Here, it is to be mentioned that the transverse component of internal momentum of the pseudoscalar meson ${ }^{1}$ can be expressed as $\hat{q}^{\prime}=\hat{q}+\hat{m}_{2} \hat{P}^{\prime}$, as in Eq. (14), where $\hat{m}_{2}$ act as momentum partitioning parameters. Now squaring both sides of Eq. (14) that connect $\hat{q}^{\prime}$ with $\hat{q}$, making use of the fact that $\hat{P}^{\prime}$ and $\hat{q}$ are both transverse to the initial hadron momentum, and $\left|\hat{P}^{\prime}\right|=|\vec{P}|=\frac{M^{2}-M^{\prime 2}}{2 M}$, we can express the relationship between $\hat{q}^{2}$ and $\hat{q}^{2}$ as

$\hat{q}^{\prime 2}=\hat{q}^{2}+2 \hat{m}_{2} \frac{\left(M^{2}-M^{\prime 2}\right)}{2 M}|\hat{q}|+\hat{m}_{2}^{2} \frac{\left(M^{2}-M^{\prime 2}\right)^{2}}{4 M^{2}}$

where, $|\hat{q}|$ is the length of the $3 \mathrm{D}$ vector $\hat{q}$, defined as $|\hat{q}|=\sqrt{\hat{q}^{2}}=\sqrt{q^{2}-(q \cdot P)^{2} / P^{2}}$, and is a Lorentz-invariant variable.

\footnotetext{
${ }^{1}$ Now, for the final meson, we can define the internal momentum $q^{\prime}=\left(\hat{q}^{\prime \prime}, i M^{\prime} \sigma^{\prime \prime}\right)$, where $\hat{q}^{\prime \prime}=q^{\prime}-\sigma^{\prime \prime} P^{\prime}$ is a component of $q^{\prime}$ that is transverse to $P^{\prime}$ and $\sigma^{\prime \prime}=q^{\prime} \cdot P^{\prime} / P^{\prime 2}$ is the component of $q^{\prime}$ that is longitudinal to $P^{\prime}$, such that $P^{\prime} \cdot \hat{q}^{\prime \prime}=0$, regardless of whether $P^{\prime} \cdot q^{\prime}=0$ or $P^{\prime} \cdot q^{\prime} \neq 0$. Here $\hat{q}^{\prime \prime 2}=q^{\prime 2}-$ $\left(P^{\prime} \cdot q^{\prime}\right)^{2} / P^{\prime 2}$ is Lorentz invariant and a four-scalar having validity over the entire 4D space and in the rest frame of the final hadron (i.e., $P^{\prime} \cdot q^{\prime}=0$, which is the same as instantaneous approximation) $q^{\prime}=\left(\hat{q}^{\prime \prime}, i 0\right)$; these wave functions reduce to $\phi\left(\vec{q}^{\prime \prime}\right)$. But for transition amplitude calculations, we choose to calculate in the initial hadron rest frame. For this, we need to perform Lorentz transformation, where the internal momentum of the final hadron is written as $q^{\prime}=\left(\hat{q}^{\prime}, i M \sigma^{\prime}\right)$. The $3 \mathrm{D}$ vector $\hat{q}^{\prime}=q^{\prime}-\sigma^{\prime} P$ is transverse to $P$ [where $\sigma^{\prime}=q^{\prime} \cdot P / P^{2}$ (see Sec. III)], and $\hat{q}^{\prime 2}$ is a Lorentz-invariant quantity and a four-scalar. Here $q^{\prime}$ acquires the time component $\sigma^{\prime}$ that is transformed to $\sigma$ through Eq. (17), which is later integrated out to obtain $M_{f i}$ in Eq. (31) through pole integrations in a complex $\sigma$ plane. However, as explained after Eq. (50), the wave functions of P-wave or S-wave mesons in a final state will not involve the time component $\sigma^{\prime}$ due to their dependence on $\left|\hat{q}^{\prime}\right|$ and/or even powers of $\hat{q}^{\prime}$, which are both Lorentz-invariant variables.
} 
The transition amplitude $M_{f i}$ is expressed as

$$
M_{f i}=F_{V P} \epsilon_{\mu \nu \alpha \beta} P_{\mu} \epsilon_{\nu}^{\lambda^{\prime}} \epsilon_{\alpha}^{\lambda} P_{\beta}^{\prime},
$$

where the antisymmetric tensor $\epsilon_{\mu \nu \alpha \beta}$ ensures its gauge invariance. Here, $F_{V P}$ is the transition form factor for $V \rightarrow P \gamma$, with the following expression:

$$
\begin{aligned}
F_{V P}= & -e N_{P} N_{V} \frac{M^{\prime}}{M^{3}} \int \frac{d^{3} \hat{q}}{(2 \pi)^{3}} \frac{\phi_{P}\left(\hat{q}^{\prime}\right) \phi_{V}(\hat{q})}{16 \omega_{1} \omega_{2} \omega_{1}^{\prime} \omega_{2}^{\prime}}\left[T_{1}-T_{2} \frac{M^{2}-M^{\prime 2}}{2 M M^{\prime 2}}|\hat{q}|\right], \\
T_{1}= & 4\left(\alpha_{1}+\alpha_{2}+\alpha_{3}+\alpha_{4}\right) M^{2}\left(m_{1}-m_{2}\right) \hat{m}_{2}\left(-\hat{q}^{2}-\omega_{1} \omega_{2}-m_{1} m_{2}\right) \\
& -4\left(\alpha_{1}-\alpha_{2}-\alpha_{3}+\alpha_{4}\right) M^{2} \hat{m}_{2}\left(\omega_{1} m_{2}+\omega_{2} m_{1}\right)\left(\omega_{1}^{\prime}+\omega_{2}^{\prime}\right), \\
T_{2}= & 4\left(\alpha_{1}+\alpha_{2}+\alpha_{3}+\alpha_{4}\right) M^{2}\left(m_{1}-m_{2}\right)\left(\omega_{1}^{\prime} \omega_{2}^{\prime}-\omega_{1} \omega_{2}-M\left(\omega_{1}^{\prime}+\omega_{2}^{\prime}\right) \hat{m}_{2} \frac{M^{2}+M^{\prime 2}}{M^{2}}\right) \\
& \left.-4\left(\alpha_{1}-\alpha_{2}-\alpha_{3}+\alpha_{4}\right) M^{2}\left(\omega_{1}^{\prime} m_{2}+\omega_{2}^{\prime} m_{1}\right)\left(\omega_{1}+\omega_{2}\right)+\left(\omega_{1} m_{2}+\omega_{2} m_{1}\right)\left(\omega_{1}^{\prime}+\omega_{2}^{\prime}\right)\right) \\
& +4\left(\alpha_{1}-\alpha_{2}+\alpha_{3}-\alpha_{4}\right) M\left(m_{1}-m_{2}\right) \hat{m}_{2}\left(\omega_{1}+\omega_{2}\right) \frac{M^{2}+M^{\prime 2}}{2} .
\end{aligned}
$$

The above expression corresponds to $F_{V P}\left(k^{2}=0\right)$, which corresponds to an emission of a real photon. However, since in this work we were mainly interested in the calculation of decay widths for various transitions, detailed calculations of $F_{V P}\left(k^{2}\right)$ along the lines of $[10,16]$ will be relegated to a separate paper. Now we proceed to calculate the decay widths for the process $V->P \gamma$, which corresponds to an emission of a real photon, for which we need $F_{V P}\left(k^{2}=0\right)$ given above. The kinematical relation connecting $\hat{q}^{\prime 2}$ with $\hat{q}^{2}$ is given in Eq. (40). To calculate the decay widths, we need to calculate the spin averaged amplitude square $\left|\bar{M}_{f i}\right|^{2}$, where $\left|\bar{M}_{f i}\right|^{2}=\frac{1}{2 j+1} \sum_{\lambda, \lambda^{\prime}}\left|M_{f i}\right|^{2}$, where we average over the initial polarization states $\lambda$ of the $V$ meson, and sum over the final polarization $\lambda^{\prime}$ of a photon. We make use of the normalizations, $\Sigma_{\lambda} \epsilon_{\mu}^{\lambda} \epsilon_{\nu}^{\lambda}=\frac{1}{3}\left(\delta_{\mu \nu}+\frac{P_{\mu} P_{\nu}}{M^{2}}\right)$ for the vector meson and $\Sigma_{\lambda^{\prime}} \epsilon_{\mu}^{\lambda^{\prime}} \epsilon_{\nu}^{\lambda^{\prime}}=\delta_{\mu \nu}$, for the emitted photon, with $M_{f i}$ taken from Eq. (41).

The spin-averaged amplitude square of the process, obtained after dividing by the total spin states $(2 j+1)$ of the initial vector meson, can be obtained as

$$
\left|\overline{M_{f i}}\right|^{2}=-\frac{2 e^{2}}{3}\left[M^{2} M^{\prime 2}-\left(P . P^{\prime}\right)^{2}\right]\left|F_{V P}(0)\right|^{2} .
$$

In the above equation, we evaluate $P \cdot P^{\prime}=-M E^{\prime}$ in the rest frame of the initial vector meson, where $E^{\prime}=$ $\sqrt{\vec{P}^{\prime 2}+M^{\prime 2}}$ is the energy of the final pseudoscalar meson, giving $P . P^{\prime}=-\left(\frac{M^{2}+M^{\prime 2}}{2}\right)$. Thus, $\left|\bar{M}_{f i}\right|^{2}$ can be expressed as

$$
\left|\bar{M}_{f i}\right|^{2}=\frac{2}{3} e^{2} \frac{\left(M^{2}-M^{\prime 2}\right)^{2}}{4}\left|F_{V P}(0)\right|^{2} .
$$

The decay width of the process $(V \rightarrow P \gamma)$ in the rest frame of the initial vector meson is expressed as

$$
\Gamma_{V \rightarrow P \gamma}=\frac{\left|\bar{M}_{f i}\right|^{2}}{8 \pi M^{2}}\left|\overrightarrow{P^{\prime}}\right|
$$

where we make use of the fact that the modulus of the momentum of the emitted pseudoscalar meson can be expressed in terms of masses of particles as $\left|\overrightarrow{P^{\prime}}\right|=|\vec{k}|=$ $\omega_{k}=\frac{1}{2 M}\left(M^{2}-M^{\prime 2}\right)$, where $\omega_{k}$ is the kinematically allowed energy of the emitted photon. Thus, $\Gamma$ in turn can be expressed as

$$
\Gamma=\frac{\alpha_{e . m .}}{3}\left|F_{V P}\right|^{2} \omega_{k}^{3} .
$$

We now calculate the radiative decay widths for the process $V \rightarrow S+\gamma$ in the next section.

\section{RADIATIVE DECAYS OF HEAVY-LIGHT QUARKONIA THROUGH $V \rightarrow S \gamma$}

$E 1$ transitions always involve excited states. The scattering amplitude of the decay process $V \rightarrow S \gamma$ can be written as 


$$
\begin{aligned}
M_{f i}= & -i e \int \frac{d^{3} \hat{q}}{(2 \pi)^{3}} \frac{1}{M^{2}} \operatorname{Tr}\left[\alpha_{1} \not p \bar{\psi}_{S}^{++}\left(\hat{q}^{\prime}\right) \phi^{\prime} \psi_{V}^{++}(\hat{q})\right. \\
& +\alpha_{2} \not P \bar{\psi}_{S}^{++}\left(\hat{q}^{\prime}\right) \phi^{\prime \prime} \psi_{V}^{--}(\hat{q})+\alpha_{3} \not P \bar{\psi}_{S}^{--}\left(\hat{q}^{\prime}\right) \phi^{\prime \prime} \psi_{V}^{++}(\hat{q}) \\
& \left.+\alpha_{4} \not P \bar{\psi}_{S}^{--}\left(\hat{q}^{\prime}\right) \phi^{\prime \prime} \psi_{V}^{--}(\hat{q})\right] .
\end{aligned}
$$

After the 3D reduction of the 4D BS wave function of a scalar meson under a CIA, we express the 3D BS wave function with dimensionality $M$ as

$\psi_{S}(\hat{q})=N_{S}\left[M f_{1}(\hat{q})+i \not p f_{2}(\hat{q})-i \hat{q} f_{3}(\hat{q})+2 \frac{\not P \hat{q}}{M} f_{4}(\hat{q})\right]$.

Making use of the fact that the most leading Dirac structure in a scalar meson BS wave function is $M I$ ( $I$ being the unit $4 \times 4$ unit matrix), and making use of [18], we express the $3 \mathrm{D}$ scalar meson BS wave function as

$$
\psi_{S}(\hat{q})=N_{S}\left(M^{\prime}\right) \phi_{S}\left(\hat{q}^{\prime}\right),
$$

where $\phi_{S}(\hat{q})$ is the spatial part of this wave function, whose analytic forms obtained by power series solutions of 3D mass spectral equations [derived from 3D Salpeter equations in Eq. (6)], in the variable $\hat{q}$ (which is in fact $|\hat{q}|$ ) for a $\mathrm{P}$-wave meson in its own rest frame, calculated in [18] are

$$
\begin{aligned}
& \phi_{S}(1 P, \hat{q})=\sqrt{\frac{2}{3}} \frac{1}{\pi^{3 / 4}} \frac{1}{\beta_{S}^{5 / 2}} \hat{q} e^{-\frac{\hat{q}^{2}}{2 \beta_{S}^{2}}}, \\
& \phi_{S}(2 P, \hat{q})=\sqrt{\frac{5}{3}} \frac{1}{\pi^{3 / 4}} \frac{1}{\beta_{S}^{5 / 2}} \hat{q}\left(1-\frac{2 \hat{q}^{2}}{5 \beta_{S}^{2}}\right) e^{-\frac{\hat{q}^{2}}{2 \beta_{S}^{2}}}, \\
& \phi_{S}(3 P, \hat{q})=\sqrt{\frac{35}{12}} \frac{1}{\pi^{3 / 4}} \frac{1}{\beta_{S}^{5 / 2}} \hat{q}\left(1-\frac{4 \hat{q}^{2}}{5 \beta_{S}^{2}}+\frac{4 \hat{q}^{4}}{35 \beta_{S}^{4}}\right) e^{-\frac{\hat{q}^{2}}{2 \beta_{S}^{2}}}, \\
& \phi_{S}(4 P, \hat{q})=\sqrt{\frac{35}{8}} \frac{1}{\pi^{3 / 4}} \frac{1}{\beta_{S}^{5 / 2}} \hat{q}\left(1-\frac{6 \hat{q}^{2}}{5 \beta_{S}^{2}}+\frac{12 \hat{q}^{4}}{35 \beta_{S}^{4}}-\frac{8 \hat{q}^{6}}{315 \beta_{S}^{6}}\right) e^{-\frac{\hat{q}^{2}}{2 \beta_{S}^{2}}} .
\end{aligned}
$$

These wave functions in Eq. (50) involve even powers of $\hat{q}$ along with odd power $\hat{q}$. Here $\hat{q}=|\hat{q}|$ as explained above is the length of the $3 \mathrm{D}$ vector $\hat{q}$ and is expressed as $|\hat{q}|=\sqrt{q^{2}-(q \cdot P)^{2} / P^{2}}$. It is a Lorentz-invariant quantity [24], along with even powers of $\hat{q}$, such as $\hat{q}^{2}, \hat{q}^{4}, \ldots$ etc. which are again Lorentz invariant. While for $\mathrm{S}$-wave mesons, the wave functions are only functions of even powers of $\hat{q}$. Thus when $\mathrm{P}$-wave or S-wave mesons are in the final state, their wave functions after Lorentz transformation would involve the variables $\left|\hat{q}^{\prime}\right|$ and/or even powers of $\hat{q}^{\prime}$. We express $\hat{q}^{\prime 2}$ in terms of $\hat{q}^{2}$ directly through Eq. (40), that connects $\hat{q}^{\prime 2}$ with $\hat{q}^{2}$, while odd power $\left|\hat{q}^{\prime}\right|$ is expressed as $\left|\hat{q}^{\prime}\right|=\sqrt{\hat{q}^{\prime 2}}$, where we again make use of Eq. (40). Thus as mentioned in footnote 1, the time component $\sigma^{\prime}$ of $q^{\prime}$ will not appear in the wave functions of both $\mathrm{P}$-wave and $\mathrm{S}$-wave mesons in a final state in the transition amplitude calculation.

The BS normalizer of a scalar meson $N_{S}$ can be obtained by solving the current conservation conditions and is expressed as

$$
N_{S}^{-2}=4 \hat{m}_{1} \hat{m}_{2} M^{\prime 2} \frac{1}{m_{1}} \int \frac{d^{3} \hat{q}}{(2 \pi)^{3}} \phi_{S}^{2}\left(\hat{q}^{\prime}\right) .
$$

We now obtain the ++ and -- components of the scalar meson wave function through Eq. (38) as given in Eq. (A9) with the corresponding adjoint wave functions in Eq. (A10). The expressions for,++++++-- , --++ , and ---- terms of the scattering amplitude in Eq. (31) is relegated to Appendix A. 2.

We first evaluate the trace over the gamma matrices in Eq. (47). We make use of the fact that $\hat{q}^{\prime}=\hat{q}+\hat{m}_{2} \hat{P}^{\prime}$, where $\hat{P}^{\prime}=P^{\prime}-\frac{P^{\prime} \cdot P}{P^{2}} P$. We combine various terms and further make use of the fact that for the initial vector meson $P . \epsilon^{\lambda}=0$, and in its rest frame $P^{\prime} \cdot \epsilon^{\lambda^{\prime}}=0$ (where $\epsilon^{\lambda^{\prime}}$ is the photon polarization vector). Due to this, we express $\hat{P}^{\prime} . \epsilon=$ $P^{\prime} . \epsilon$ and $\hat{P}^{\prime} . \epsilon^{\prime}=\beta P . \epsilon^{\prime}$, where $\beta=-\frac{P^{\prime} . P}{P^{2}}=-\frac{M^{2}+M^{\prime 2}}{2 M^{2}}$ from Eq. (15). We can then express the invariant matrix element $M_{f i}$ as

$$
\begin{gathered}
M_{f i}=-i e N_{S} N_{V} \frac{1}{M^{2}} \int \frac{d^{3} \hat{q}}{(2 \pi)^{3}} \frac{\phi_{S}\left(\hat{q}^{\prime}\right) \phi_{V}(\hat{q})}{16 \omega_{1} \omega_{2} \omega_{1}^{\prime} \omega_{2}^{\prime}}\left[\Theta_{1}\left(\epsilon^{\lambda^{\prime}} \cdot \epsilon^{\lambda}\right)+\Theta_{2} \beta\left(\epsilon^{\lambda^{\prime}} . P\right)\left(\epsilon^{\lambda} \cdot P^{\prime}\right)\right], \\
\Theta_{1}=4 M^{3}\left(\left(\alpha_{1}-\alpha_{2}+\alpha_{3}-\alpha_{4}\right)\left(\omega_{1}^{\prime} \omega_{2}^{\prime}-m_{1} m_{2}+\hat{q}^{\prime 2}\right)\left(\omega_{1} m_{2}+m_{1} \omega_{2}\right)\right. \\
+\left(\alpha_{1}+\alpha_{2}-\alpha_{3}-\alpha_{4}\right)\left(\omega_{1}^{\prime} m_{2}-m_{1} \omega_{2}^{\prime}\right)\left(\omega_{1} \omega_{2}+m_{1} m_{2}+\hat{q}^{2}\right)+\left[\left(\alpha_{1}+\alpha_{2}-\alpha_{3}-\alpha_{4}\right)\left(m_{1}-m_{2}\right)\left(\omega_{1}^{\prime}+\omega_{2}^{\prime}\right)\right. \\
\left.\left.-\left(\alpha_{1}-\alpha_{2}+\alpha_{3}-\alpha_{4}\right)\left(m_{1}+m_{2}\right)\left(\omega_{1}-\omega_{2}\right)\right]\left(\hat{q}^{2}+\hat{m}_{2} \frac{M^{2}-M^{\prime 2}}{2 M}|\hat{q}|\right)\right),
\end{gathered}
$$




$$
\begin{aligned}
\Theta_{2}= & \frac{16 M^{5} \hat{q}^{2}}{\left(M^{2}-M^{\prime 2}\right)^{2}}\left(\left(\alpha_{1}+\alpha_{2}-\alpha_{3}-\alpha_{4}\right)\left[-\left(\omega_{1}^{\prime} m_{2}-m_{1} \omega_{2}^{\prime}\right)+m_{2}\left(\omega_{1}^{\prime}+\omega_{2}^{\prime}\right)\right]+\left(\alpha_{1}-\alpha_{2}+\alpha_{3}-\alpha_{4}\right) \omega_{2}\left(m_{1}+m_{2}\right)\right) \\
& +\frac{8 M^{4}|\hat{q}|}{\left(M^{2}-M^{\prime 2}\right)} \hat{m}_{2}\left(-\left(\alpha_{1}+\alpha_{2}-\alpha_{3}-\alpha_{4}\right)\left(m_{1}-m_{2}\right)\left(\omega_{1}^{\prime}+\omega_{2}^{\prime}\right)+\left(\alpha_{1}-\alpha_{2}+\alpha_{3}-\alpha_{4}\right)\left(m_{1}+m_{2}\right)\left(\omega_{1}+\omega_{2}\right)\right) .
\end{aligned}
$$

After carrying out the integrals $d^{3} \hat{q}$ over $\Theta_{1}$ and $\Theta_{2}$ in Eq. (45), we can express the amplitude $M_{f i}$ as

$$
\begin{aligned}
M_{f i} & =S_{1}\left(\epsilon^{\lambda^{\prime}} \cdot \epsilon^{\lambda}\right)+S_{2} \beta\left(\epsilon^{\lambda^{\prime}} \cdot P\right)\left(\epsilon^{\lambda} \cdot P^{\prime}\right), \\
S_{1} & =-i e N_{S} N_{V} \frac{1}{M^{2}} \int \frac{d^{3} \hat{q}}{(2 \pi)^{3}} \frac{\phi_{S}\left(\hat{q}^{\prime}\right) \phi_{V}(\hat{q})}{16 \omega_{1} \omega_{2} \omega_{1}^{\prime} \omega_{2}^{\prime}} \Theta_{1}, \\
S_{2} & =-i e N_{S} N_{V} \frac{1}{M^{2}} \int \frac{d^{3} \hat{q}}{(2 \pi)^{3}} \frac{\phi_{S}\left(\hat{q}^{\prime}\right) \phi_{V}(\hat{q})}{16 \omega_{1} \omega_{2} \omega_{1}^{\prime} \omega_{2}^{\prime}} \Theta_{2} .
\end{aligned}
$$

And, $S_{1}$ and $S_{2}$ are the form factors. Now, to calculate the decay widths, we need to calculate the spin averaged amplitude square $\left|\bar{M}_{f i}\right|^{2}$, where $\left|\bar{M}_{f i}\right|^{2}=\frac{1}{2 j+1} \sum_{\lambda, \lambda^{\prime}}\left|M_{f i}\right|^{2}$, where we average over the initial polarization states $\lambda$ of the $V$ meson, and sum over the final polarization $\lambda^{\prime}$ of the photon. We make use of the normalizations $\Sigma_{\lambda} \epsilon_{\mu}^{\lambda} \epsilon_{\nu}^{\lambda}=\frac{1}{3}\left(\delta_{\mu \nu}+\frac{P_{\mu} P_{\nu}}{M^{2}}\right)$ for the vector meson and $\Sigma_{\lambda^{\prime}} \epsilon_{\mu}^{\lambda^{\prime}} \epsilon_{\nu}^{\lambda^{\prime}}=\delta_{\mu \nu}$ for the emitted photon, with $M_{f i}$ taken from the previous equations. This gives $\sum_{\lambda^{\prime}} \sum_{\lambda}\left|\epsilon^{\lambda^{\prime}} \cdot \epsilon^{\lambda}\right|^{2}=1$.

The spin-averaged amplitude square of the process can be written as
$\left|\bar{M}_{f i}\right|^{2}=\frac{1}{3}\left[\left|S_{1}\right|^{2}+\frac{1}{3} \beta^{2}\left[M^{2} M^{\prime 2}-\left(P^{\prime} . P\right)^{2}\right]\left|S_{2}\right|^{2}\right]$,

where $\beta^{2}=\frac{\left(M^{2}+M^{2}\right)^{2}}{4 M^{2}}$. We can write the decay width,

$$
\Gamma_{V \rightarrow S \gamma}=\frac{\left|\bar{M}_{f i}\right|^{2}}{8 \pi M^{2}}\left|\overrightarrow{P^{\prime}}\right|
$$

where we make use of the fact that the modulus of the momentum of the emitted pseudoscalar meson can be expressed in terms of masses of particles as $\left|\overrightarrow{P^{\prime}}\right|=\frac{1}{2 M}\left(M^{2}-M^{\prime 2}\right)$.

\section{RADIATIVE DECAYS OF HEAVY-LIGHT QUARKONIA THROUGH $S \rightarrow V \gamma$}

We proceed to evaluate the process in the same manner as $V \rightarrow S \gamma$, using Fig. 1, where the initial scalar meson decays into a vector meson and a photon. Drawing analogy from $V \rightarrow P \gamma$, and $V \rightarrow S \gamma$, the effective 3D form of the transition amplitude $M_{f i}$ for $S \rightarrow V \gamma$ under a covariant instantaneous ansatz can be expressed as

$$
\begin{aligned}
M_{f i}= & -i e \int \frac{d^{3} \hat{q}}{(2 \pi)^{3}} \frac{1}{M^{2}} \operatorname{Tr}\left[\alpha_{1} \not P \bar{\psi}_{V}^{++}\left(\hat{q}^{\prime}\right) \phi^{\prime \prime} \psi_{S}^{++}(\hat{q})+\alpha_{2} \not \bar{\psi}_{V}^{++}\left(\hat{q}^{\prime}\right) \phi^{\prime \prime} \psi_{S}^{--}(\hat{q})\right. \\
& \left.+\alpha_{3} \not \bar{\psi}_{V}^{--}\left(\hat{q}^{\prime}\right) \phi^{\prime} \psi_{S}^{++}(\hat{q})+\alpha_{4} \not P \bar{\psi}_{V}^{--}\left(\hat{q}^{\prime}\right) \phi^{\prime \prime} \psi_{S}^{--}(\hat{q})\right] .
\end{aligned}
$$

The transition amplitude of the $S \rightarrow V \gamma$ process can be obtained as

$$
M_{f i}=e N_{S} N_{V} \frac{M^{\prime}}{M^{3}} \int \frac{d^{3} \hat{q}}{(2 \pi)^{3}} \frac{\phi_{S}\left(\hat{q}^{\prime}\right) \phi_{V}(\hat{q})}{16 \omega_{1} \omega_{2} \omega_{1}^{\prime} \omega_{2}^{\prime}}[T R]
$$

where

$$
\begin{aligned}
{[T R]=} & \operatorname{Tr}\left[-\left(\alpha_{1}-\alpha_{2}+\alpha_{3}-\alpha_{4}\right) i M^{3}\left(\omega_{1}^{\prime} \omega_{2}^{\prime}+m_{1} m_{2}\right)\left(\omega_{1} m_{2}-m_{1} \omega_{2}\right) \phi^{\lambda} \phi^{\lambda^{\prime}}\right. \\
& +\left(\alpha_{1}-\alpha_{2}+\alpha_{3}-\alpha_{4}\right) i M^{3}\left(\omega_{1} m_{2}-m_{1} \omega_{2}\right) \hat{\phi}^{\prime} \phi^{\lambda} \hat{\phi}^{\prime} \phi^{\lambda^{\prime}}-\left(\alpha_{1}+\alpha_{2}+\alpha_{3}+\alpha_{4}\right) i M^{2}\left(m_{1}+m_{2}\right) \boldsymbol{p}^{\prime} \hat{\phi}^{\prime} \phi^{\lambda} \hat{\phi}^{\prime} \phi^{\lambda^{\prime}} \hat{\phi} \mid \\
& -\left(\alpha_{1}+\alpha_{2}-\alpha_{3}-\alpha_{4}\right) i M^{3}\left(\omega_{1}^{\prime} m_{2}+m_{1} \omega_{2}^{\prime}\right)\left(\omega_{1} \omega_{2}-m_{1} m_{2}+\hat{q}^{2}\right) \phi^{\lambda} \phi^{\lambda^{\prime}} \\
& -\left(\alpha_{1}-\alpha_{2}+\alpha_{3}-\alpha_{4}\right) i M^{3}\left(m_{1}\left(\omega_{1}+\omega_{2}\right) \hat{\phi}^{\prime} \phi^{\lambda} \phi^{\lambda^{\prime}} \hat{\phi}+m_{2}\left(\omega_{1}+\omega_{2}\right) \phi^{\lambda} \hat{\phi}^{\prime} \phi^{\lambda^{\prime}} \hat{\phi}\right) \\
& \left.+\left(\alpha_{1}+\alpha_{2}-\alpha_{3}-\alpha_{4}\right) i M^{3}\left(m_{1}+m_{2}\right)\left(\omega_{1}^{\prime} \hat{\phi}^{\prime} \phi^{\lambda} \phi^{\lambda \lambda^{\prime}} \hat{\phi}-\omega_{2}^{\prime} \phi^{\lambda} \hat{\phi}^{\prime} \phi^{\lambda^{\prime}} \hat{\phi}\right)\right] .
\end{aligned}
$$

We first evaluate the trace over the gamma matrices in the above equation. We make use of the fact that $\hat{q}^{\prime}=\hat{q}+\hat{m}_{2} \hat{P}^{\prime}$, where $\hat{P}^{\prime}=P^{\prime}-\frac{P^{\prime} . P}{P^{2}} P$. We combine various terms and further make use of the fact that for the initial scalar meson at rest in 
its own frame, $P . \epsilon^{\lambda^{\prime}}=0$ and $P^{\prime} \cdot \epsilon^{\lambda}=0$, where $\epsilon^{\lambda}$ is the polarization vector of the emitted vector meson with momentum $P^{\prime}$ and $\epsilon^{\lambda^{\prime}}$ is the photon polarization vector. Due to this, we express $\hat{P}^{\prime} . \epsilon=\beta(P . \epsilon)$ and $\hat{P}^{\prime} \cdot \epsilon^{\prime}=P^{\prime} . \epsilon^{\prime}$, where $\beta=-\frac{P^{\prime} \cdot P}{P^{2}}=-\frac{M^{2}+M^{\prime 2}}{2 M^{2}}$. We can then express the invariant matrix element $M_{f i}$ as

$$
M_{f i}=-i e N_{S} N_{V} \frac{1}{M^{2}} \int \frac{d^{3} \hat{q}}{(2 \pi)^{3}} \frac{\phi_{S}\left(\hat{q}^{\prime}\right) \phi_{V}(\hat{q})}{16 \omega_{1} \omega_{2} \omega_{1}^{\prime} \omega_{2}^{\prime}}\left[\Theta_{1}^{\prime}\left(\epsilon^{\lambda^{\prime}} \cdot \epsilon^{\lambda}\right)+\Theta_{2}^{\prime} \beta\left(\epsilon^{\lambda} \cdot P\right)\left(\epsilon^{\lambda^{\prime}} \cdot P^{\prime}\right)\right]
$$

where

$$
\begin{aligned}
\Theta_{1}{ }^{\prime}= & 4\left(\alpha_{1}-\alpha_{2}+\alpha_{3}-\alpha_{4}\right) M^{3}\left(\left(-m_{2} \omega_{1}+m_{1} \omega_{2}\right)\left(m_{1} m_{2}+\omega_{1}{ }^{\prime} \omega_{2}{ }^{\prime}+\hat{q}^{\prime 2}\right)\right. \\
& \left.+\frac{1}{2 M}\left[\left(\hat{m}_{2}\left(M^{2}-M^{\prime 2}\right)|\hat{q}|+2 M \hat{q}^{2}\right)\left(m_{1}-m_{2}\right)\left(\omega_{1}+\omega_{2}\right)\right]\right)-4 M^{3}\left(\alpha_{1}+\alpha_{2}-\alpha_{3}-\alpha_{4}\right) \\
& \times\left(\left(\omega_{1} \omega_{2}-m_{1} m_{2}+\hat{q}^{2}\right)\left(m_{2} \omega_{1}^{\prime}+m_{1} \omega_{2}^{\prime}\right)+\frac{1}{2 M}\left(\hat{m}_{2}\left(M^{2}-M^{\prime 2}\right)|\hat{q}|+2 M \hat{q}^{2}\right)\left(m_{1}+m_{2}\right)\left(\omega_{1}^{\prime}+\omega_{2}^{\prime}\right)\right) ; \\
\Theta_{2}^{\prime}= & \left(\alpha_{1}-\alpha_{2}+\alpha_{3}-\alpha_{4}\right) M^{3}\left(\hat{m}_{2}^{2}\left(m_{2} \omega_{1}-m_{1} \omega_{2}\right)-32 \frac{M^{2}}{\left(M^{2}-M^{\prime 2}\right)^{2}}\left(m_{1}+m_{2}\right) \omega_{2} \hat{q}^{2}\right. \\
& \left.-16 \frac{M \hat{m}_{2}}{M^{2}-M^{\prime 2}}|\hat{q}|\left(-m_{2} \omega_{1}+2 m_{1} \omega_{2}+m_{2} \omega_{2}\right)\right) \\
& +\left(\alpha_{1}+\alpha_{2}-\alpha_{3}-\alpha_{4}\right) M^{3}\left(-\frac{32 M^{2}}{\left(M^{2}-M^{\prime 2}\right)^{2}}\left(m_{1}+m_{2}\right) \omega_{2} \hat{q}^{2}-16 \frac{M \hat{m}_{2}}{\left(M^{2}-M^{\prime 2}\right)}|\hat{q}|\left(m_{1}+m_{2}\right) \omega_{2}{ }^{\prime}\right) .
\end{aligned}
$$

Thus, $M_{f i}$ can be expressed as

$$
\begin{aligned}
M_{f i} & =S_{1}^{\prime}\left(\epsilon^{\lambda^{\prime}} \cdot \epsilon^{\lambda}\right)+S_{2}^{\prime} \beta\left(\epsilon^{\lambda^{\prime}} \cdot P^{\prime}\right)\left(\epsilon^{\lambda} \cdot P\right), \\
S_{1}^{\prime} & =-i e N_{S} N_{V} \frac{1}{M^{2}} \int \frac{d^{3} \hat{q}}{(2 \pi)^{3}} \frac{\phi_{S}\left(\hat{q}^{\prime}\right) \phi_{V}(\hat{q})}{16 \omega_{1} \omega_{2} \omega_{1}^{\prime} \omega_{2}^{\prime}} \Theta_{1}{ }^{\prime}, \\
S_{2}^{\prime} & =-i e N_{S} N_{V} \frac{1}{M^{2}} \int \frac{d^{3} \hat{q}}{(2 \pi)^{3}} \frac{\phi_{S}\left(\hat{q}^{\prime}\right) \phi_{V}(\hat{q})}{16 \omega_{1} \omega_{2} \omega_{1}^{\prime} \omega_{2}^{\prime}} \Theta_{2}{ }^{\prime} .
\end{aligned}
$$

To calculate the decay widths, we again need to calculate the spin averaged amplitude square $\left|\bar{M}_{f i}\right|^{2}$, where $\left|\bar{M}_{f i}\right|^{2}=\sum_{\lambda, \lambda^{\prime}}\left|M_{f i}\right|^{2}$, where we sum over the final polarization states $\lambda^{\prime}$ of the photon and $\lambda$ of the $V$ meson.

The spin averaged amplitude modulus square gives

$$
\left|\bar{M}_{f i}\right|^{2}=\left[\left|S_{1}^{\prime}\right|^{2}+\frac{1}{3} \beta^{2}\left[P^{2} P^{\prime 2}-\left(P^{\prime} . P\right)^{2}\right]\left|S_{2}^{\prime}\right|^{2}\right] .
$$

The decay widths $\Gamma$ for the process $S \rightarrow V \gamma$ are given by Eq. (56), with $P^{\prime}$ now the momentum of the emitted vector meson.

\section{RESULTS AND DISCUSSION}

We have studied radiative decays of conventional heavylight quarkonia through $M 1$ and $E 1$ transitions in the framework of the Bethe-Salpeter equation. Such processes involve quark-triangle diagrams and two hardon-quark vertices and are difficult to evaluate in a BSE under a CIA. In this work we have given a generalized method of handling quark triangle diagrams with two hadron-quark vertices in the framework of a $4 \times 4$ BSE by expressing the transition amplitude $M_{f i}$ [Eqs. (31) and (32)] as a linear superposition of terms involving all possible combinations of ++ and -- components of Salpeter wave functions of final and initial hadrons, through not only the terms ++ ++ and ---- but also the terms like ++-- and --++ , with each of the four terms being associated with a coefficient $\alpha_{i}(i=1, \ldots, 4)$, which is the result of pole integration in the complex $\sigma$ plane, with pole positions in Eq. (27) (shown in Fig. 2). This superposition of all possible terms in Eqs. (31) and (32) should be a feature of relativistic frameworks.

Using this generalized expression for $M_{f i}$ in Eqs. (31) and (32), we have evaluated the decay widths for $M 1$ transitions, ${ }^{3} S_{1} \rightarrow{ }^{1} S_{0}+\gamma$, involving the decays of the ground and excited states of the heavy-light mesons such as $B_{c}^{*}, B^{*}, J / \Psi, D^{*}$, and $D_{s}^{*}$. In regard to the $E 1$ transitions, we have studied the processes ${ }^{3} S_{1} \rightarrow{ }^{1} P_{0}+\gamma$ that involve the decays of $\Psi(2 S), B_{c}^{*}(2 S)$, and $D^{*}(2 S)$, and the processes ${ }^{1} P_{0} \rightarrow{ }^{3} S_{1}+\gamma$ that involve decays of $\chi_{c 0}(1 P), B_{c}(1 P)$ and $B_{c}(2 P)$.

We used algebraic forms of the 3D Salpeter wave functions obtained through analytic solutions of mass spectral equations in an approximate harmonic oscillator 
TABLE I. Radiative decay widths of heavy-light mesons (in keV) for $M 1$ transitions in BSE, along with experimental data and results of other models.

\begin{tabular}{|c|c|c|c|c|c|}
\hline & BSE-CIA & Experiment & LFQM & $\mathrm{PM}$ & RQM \\
\hline$\Gamma_{J / \psi\left(1 S_{1}\right) \rightarrow \eta_{c}}\left(1 S_{0}\right) \gamma$ & 1.7036 & $1.5793 \pm 0.0112[35]$ & $1.69 \pm 0.05[11]$ & $1.8[36]$ & $1.050[37]$ \\
\hline$\Gamma_{\psi\left(2 S_{1}\right) \rightarrow \eta_{c}\left(2 S_{0}\right) \gamma}$ & 0.18204 & $0.2002 \pm 0.008[38]$ & & $0.4[36]$ & \\
\hline$\Gamma_{\psi\left(2 S_{1}\right) \rightarrow \eta_{c}\left(1 S_{0}\right) \gamma}$ & 0.9340 & 0.9724 & & & \\
\hline$\Gamma_{B_{c}^{*}\left(1 S_{1}\right) \rightarrow B_{c}\left(1 S_{0}\right) \gamma}$ & 0.0664 & & & $0.06[39]$ & $0.033[37]$ \\
\hline$\Gamma_{B_{c}^{*}\left(2 S_{1}\right) \rightarrow B_{c}\left(2 S_{0}\right) \gamma}$ & 0.0360 & & & $0.01[39]$ & $0.017[37]$ \\
\hline$\Gamma_{B_{s}^{*}\left(1 S_{1}\right) \rightarrow B_{s}\left(1 S_{0}\right) \gamma}$ & 0.0624 & $0.064 \pm 0.016[40]$ & $0.068 \pm 0.017[11]$ & & \\
\hline$\Gamma_{B_{s}^{*}\left(2 S_{1}\right) \rightarrow B_{s}}\left(2 S_{0}\right) \gamma$ & 0.04708 & & & & \\
\hline$\Gamma_{B^{*}\left(1 S_{1}\right) \rightarrow B\left(1 S_{0}\right) \gamma}$ & 0.1364 & $0.13 \pm 0.01[40]$ & $0.13 \pm 0.01[11]$ & & \\
\hline$\Gamma_{B^{*}\left(2 S_{1}\right) \rightarrow B\left(2 S_{0}\right) \gamma}$ & 0.1467 & & & & \\
\hline$\Gamma_{D_{s}^{*}\left(1 S_{1}\right) \rightarrow D_{s}\left(1 S_{0}\right) \gamma}$ & 0.2018 & & $0.17 \pm 0.01[40]$ & & $0.213[41]$ \\
\hline$\Gamma_{D^{*}\left(1 S_{1}\right) \rightarrow D\left(1 S_{0}\right) \gamma}$ & 1.2843 & $1.3344 \pm 0.0072[40]$ & $0.90 \pm 0.02[11]$ & & \\
\hline$\Gamma_{D^{*}\left(2 S_{1}\right) \rightarrow D\left(2 S_{0}\right) \gamma}$ & 0.1381 & & & & \\
\hline
\end{tabular}

basis for ground and excited states of $0^{++}, 1^{--}$, and $0^{-+}$ heavy-light quarkonia for calculation of their decay widths. The input parameters we used were $C_{0}=0.69, \omega_{0}=$ $0.22 \mathrm{GeV}, \Lambda_{\mathrm{QCD}}=0.25 \mathrm{GeV}$, and $A_{0}=0.01$, along with the input quark masses $m_{u}=0.30 \mathrm{GeV}, m_{s}=0.43 \mathrm{GeV}$, $m_{c}=1.49 \mathrm{GeV}$, and $m_{b}=4.67 \mathrm{GeV}$, which were obtained by fitting to their mass spectra [19]. We have compared our results with experimental data and other models, and found reasonable agreements.

We get reasonable agreements of our decay widths for $M 1$ transitions, $n S \rightarrow n^{\prime} S+\gamma$. This can be seen from Table I for the transitions $J / \Psi(1 S) \rightarrow \eta_{c}(1 S), \Psi(2 S) \rightarrow$ $\eta_{c}(2 S)$, and $\Psi(2 S) \rightarrow \eta_{c}(1 S)$. Similar agreements of our decay widths for $E 1$ transitions are noticed for $n S \rightarrow$ $n^{\prime} P+\gamma$ and $n P \rightarrow n^{\prime} S+\gamma$, as can be seen from Table II.

We wish to mention that $F_{V P}(0)$ in Eq. (34) are the electromagnetic coupling constants $g_{V P \gamma}$. It is seen that our coupling constant $g_{J / \Psi \eta_{c} \gamma}=0.745 \mathrm{GeV}^{-1}$ (expt. $=$ $0.570 \pm 0.110 \mathrm{GeV}^{-1}$ ) [44], while the coupling constant $g_{D * D \gamma}=-0.438 \mathrm{GeV}^{-1}$, which can be compared with experimental data that gives $-0.466 \mathrm{GeV}^{-1}$ [44] and $-0.384 \mathrm{GeV}^{-1}$ [11]. Our $g_{D_{s} * D_{s} \gamma}=-0.173 \mathrm{GeV}^{-1}$, which is comparable to the RQM model value $0.161 \mathrm{GeV}^{-1}$ [11]. Our $g_{B_{s} * B_{s} \gamma}=-0.4773 \mathrm{GeV}^{-1}$ which can be compared with -0.536 [11] and -0.657 [41]. Similarly, our $g_{B * B \gamma}=-0.764 \mathrm{GeV}^{-1}$, which can be compared with -0.749 [11] and -0.891 [41]. However, these results show that various models have a wide range of variations of coupling constants $g_{V P \gamma}$ for different transitions.

Similarly we again see a wide range of variations in different models for $M 1$ transitions, particularly for decays of $J / \Psi$ and $\Psi(2 S)$. Further, our $n S \rightarrow n S$ transitions show a marked decrease as we go from ground to higher excited

TABLE II. Radiative decay widths of heavy-light mesons (in keV) for $E 1$ transitions, along with experimental data and results of other models.

\begin{tabular}{|c|c|c|c|c|}
\hline & BSE-CIA & Experiment & PM & RQM \\
\hline$\Gamma_{\psi\left(2 S_{1}\right) \rightarrow \chi_{c 0}\left(1 P_{0}\right) \gamma}$ & 34.0419 & $28.5714 \pm 0.0432[38]$ & & 26.3 [37] \\
\hline$\Gamma_{\psi\left(3 S_{1}\right) \rightarrow \chi_{c 0}\left(2 P_{0}\right) \gamma}$ & 62.229 & & $51.4[42]$ & $65.7[36]$ \\
\hline$\Gamma_{\psi\left(3 S_{1}\right) \rightarrow \chi_{c 0}\left(1 P_{0}\right) \gamma}$ & 1.4441 & & $1.2[42]$ & \\
\hline$\Gamma_{B_{c}^{*}\left(2 S_{1}\right) \rightarrow B_{c}\left(1 P_{0}\right) \gamma}$ & 10.5249 & & $9.6[43]$ & $3.78[37]$ \\
\hline$\Gamma_{D^{*}\left(2 S_{1}\right) \rightarrow D\left(1 P_{0}\right) \gamma}$ & 1.0214 & & & \\
\hline$\Gamma_{\chi_{c 0}\left(1 P_{0}\right) \rightarrow J / \psi\left(1 S_{1}\right) \gamma}$ & 123.803 & $119.5 \pm 8[40]$ & & $161[37]$ \\
\hline$\Gamma_{\chi_{c 0}}\left(2 P_{0}\right) \rightarrow \psi\left(2 S_{1}\right) \gamma$ & 75.229 & & 68 [42] & \\
\hline$\Gamma_{\chi_{c 0}}\left(2 P_{0}\right) \rightarrow J / \psi\left(1 S_{1}\right) \gamma$ & 129.86 & & $146[42]$ & $21[36]$ \\
\hline$\Gamma_{B_{c}\left(1 P_{0}\right) \rightarrow B_{c}^{*}\left(1 S_{1}\right) \gamma}$ & 68.580 & & $65.3[39]$ & $75.5[37]$ \\
\hline$\Gamma_{B_{c}}\left(2 P_{0}\right) \rightarrow B_{c}^{*}\left(2 S_{1}\right) \gamma$ & 51.3911 & & $52.5[39]$ & 34 [37] \\
\hline
\end{tabular}


states, which is in conformity with data and other models. We have also given our predictions for radiative decays of $B_{c}^{*}(1 S), B_{c}^{*}(2 S), B_{s}^{*}(2 S), B^{*}(2 S)$, and $D^{*}(2 S)$, for which data is not yet available, and for $D_{s}^{*}(1 S)$, where PDG [40] gives only the upper limit of the decay width. In regard to $E 1$ transitions, our decay width result for $\Psi(2 S)$ is in good agreement with data, but for $\chi_{c 0}$ is higher than data, although again there is a lot of variation in results of other models. These results have been obtained using leading Dirac structures in the wave functions of $P, V$, and $S$ mesons, although incorporation of all Dirac structures is expected to give better agreement with data.

The aim of doing this study was to mainly test our analytic forms of wave functions in Eqs. (37) and (50), obtained as solutions of mass spectral equations in an approximate harmonic oscillator basis obtained analytically from a $4 \times 4$ BSE as a starting point, that has so far given good predictions [17-19] not only of the mass spectrum of heavy-light quarkonia but also their leptonic decays, twophoton and two gluon decays. The present work would in turn lead to the validation of our approach, which provides a much deeper insight than the purely numerical calculations in a $4 \times 4 \mathrm{BSE}$ approach that are prevalent in the literature.

This work mainly focused on evaluation of decay widths for $M 1$ and $E 1$ transitions. A more detailed study on not only the transition form factors of both $M 1$ and $E 1$ transitions but also the "static" form factors describing meson-photon interactions through the vertex $M \gamma M$ for various mesons will be relegated to a separate paper.

\section{ACKNOWLEDGMENTS}

The authors wish to thank Chandigarh University, and Addis Ababa University for the facilities provided during the course of this work. S. B. acknowledges discussions with K. J. S.Channey, and K. B.Vijaya Kumar. We thank the unknown referee for his valuable comments that led to the improvements in the article. E. G. would like to thank Woldia university for support to his Doctoral programme, and to Swedish International Development Agency (SIDA) for facilitating his visit to Chandigarh University during the course of this work. S. B. would thank Chandigarh University and his family for moral support during the course of this work.

\section{APPENDIX}

\section{Radiative decays through $\boldsymbol{V} \rightarrow \boldsymbol{P} \boldsymbol{\gamma}$}

Substituting the 3D BS wave function of a pseudoscalar meson in Eq. (35), we obtain the ++ and -- components as

$$
\begin{aligned}
& \psi_{P}^{++}\left(\hat{q}^{\prime}\right)=\frac{N_{P}}{4 \omega_{1}^{\prime} \omega_{2}^{\prime}} \frac{M^{\prime}}{M} \phi_{P}\left(\hat{q}^{\prime}\right)\left[M\left(\omega_{1}^{\prime} \omega_{2}^{\prime}+m_{1} m_{2}+\hat{q}^{\prime 2}\right)-i\left(\omega_{1}^{\prime} m_{2}+m_{1} \omega_{2}^{\prime}\right) \not P+i M\left(m_{1}-m_{2}\right) \hat{q}^{\prime}+\left(\omega_{1}^{\prime} \not \hat{q}^{\prime}-\omega_{2}^{\prime} \hat{q}^{\prime} \not p\right)\right] \gamma_{5}, \\
& \psi_{P}^{--}\left(\hat{q}^{\prime}\right)=\frac{N_{P}}{4 \omega_{1}^{\prime} \omega_{2}^{\prime}} \frac{M^{\prime}}{M} \phi_{P}\left(\hat{q}^{\prime}\right)\left[M\left(\omega_{1}^{\prime} \omega_{2}^{\prime}+m_{1} m_{2}+\hat{q}^{\prime 2}\right)+i\left(\omega_{1}^{\prime} m_{2}+m_{1} \omega_{2}^{\prime}\right) \not P+i M\left(m_{1}-m_{2}\right) \hat{q}^{\prime}-\left(\omega_{1}^{\prime} \not \hat{p}^{\prime}-\omega_{2}^{\prime} \hat{q}^{\prime} \not p\right)\right] \gamma_{5} .
\end{aligned}
$$

The adjoint Bethe-Salpeter wave function of a pseudoscalar meson can be obtained by evaluating $\bar{\psi}_{P}^{ \pm \pm}\left(\hat{q}^{\prime}\right)=$ $\gamma_{4}\left(\psi_{P}^{ \pm \pm}\left(\hat{q}^{\prime}\right)\right)^{+} \gamma_{4}$ as

$$
\begin{aligned}
& \bar{\psi}_{P}^{++}\left(\hat{q}^{\prime}\right)=\frac{N_{P}}{4 \omega_{1}^{\prime} \omega_{2}^{\prime}} \frac{M^{\prime}}{M} \phi_{P}\left(\hat{q}^{\prime}\right)\left[-M\left(\omega_{1}^{\prime} \omega_{2}^{\prime}+m_{1} m_{2}+\hat{q}^{2}\right)-i\left(\omega_{1}^{\prime} m_{2}+m_{1} \omega_{2}^{\prime}\right) \not P+i M\left(m_{1}-m_{2}\right) \hat{q}^{\prime}-\left(\omega_{1}^{\prime} \hat{q}^{\prime} \not P-\omega_{2}^{\prime} \not \hat{q}^{\prime}\right)\right] \gamma_{5}, \\
& \bar{\psi}_{P}^{--}\left(\hat{q}^{\prime}\right)=\frac{N_{P}}{4 \omega_{1}^{\prime} \omega_{2}^{\prime}} \frac{M^{\prime}}{M} \phi_{P}\left(\hat{q}^{\prime}\right)\left[-M\left(\omega_{1}^{\prime} \omega_{2}^{\prime}+m_{1} m_{2}+\hat{q}^{\prime 2}\right)+i\left(\omega_{1}^{\prime} m_{2}+m_{1} \omega_{2}^{\prime}\right) \not P+i M\left(m_{1}-m_{2}\right) \hat{q}^{\prime}+\left(\omega_{1}^{\prime} \hat{q}^{\prime} \not P-\omega_{2}^{\prime} \not \hat{q}^{\prime}\right)\right] \gamma_{5} .
\end{aligned}
$$

Following the same steps as in Eq. (A3), we obtain the ++ and -- components of a vector meson wave function in Eq. (35) as 
$\psi_{V}^{++}(\hat{q})=\frac{i N_{V}}{4 \omega_{1} \omega_{2}} \phi_{V}(\hat{q})\left[M\left(\omega_{1} \omega_{2}+m_{1} m_{2}\right) \phi-M \hat{\phi} \phi \hat{\phi}+i\left(\omega_{1} m_{2}+m_{1} \omega_{2}\right) \phi P P-i M\left(m_{1} \dot{\phi} \hat{\phi}+m_{2} \hat{\phi} \phi\right)+\left(\omega_{1} \phi \vec{P} \hat{\phi}-\omega_{2} \hat{\phi} \not \boldsymbol{q} \phi\right)\right]$,

$\psi_{V}^{--}(\hat{q})=\frac{i N_{V}}{4 \omega_{1} \omega_{2}} \phi_{V}(\hat{q})\left[M\left(\omega_{1} \omega_{2}+m_{1} m_{2}\right) \phi-M \hat{\phi} \phi \hat{\phi}-i\left(\omega_{1} m_{2}+m_{1} \omega_{2}\right) \phi \not P-i M\left(m_{1} \dot{\phi} \hat{\phi}+m_{2} \hat{\phi} \phi\right)-\left(\omega_{1} \phi \dot{P} \hat{\phi}-\omega_{2} \hat{\phi} \not \boldsymbol{\phi} \phi\right)\right]$,

where as the adjoint wave functions are

$$
\begin{aligned}
& \bar{\psi}_{V}^{++}(\hat{q})=\frac{-i N_{V}}{4 \omega_{1} \omega_{2}} \phi_{V}(\hat{q})\left[-M\left(\omega_{1} \omega_{2}+m_{1} m_{2}\right) \phi+M \hat{\phi} \phi \hat{\phi}-i\left(\omega_{1} m_{2}+m_{1} \omega_{2}\right) \not P \phi+i M\left(m_{1} \hat{\phi} \phi+m_{2} \phi \hat{\phi}\right)-\left(\omega_{1} \hat{\phi} \not P \phi-\omega_{2} \phi \dot{P} \hat{\phi}\right)\right],
\end{aligned}
$$

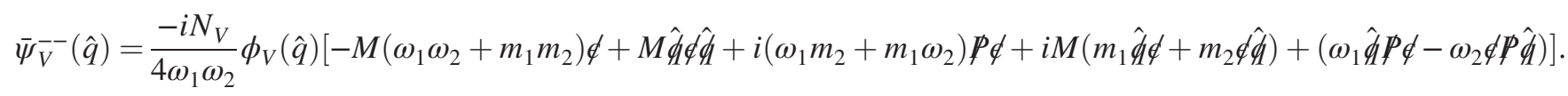

The $\not p \bar{\psi}_{P}^{++}\left(\hat{q}^{\prime}\right) \phi^{\prime \prime} \Psi_{V}^{++}(\hat{q}), \not p \bar{\psi}_{P}^{++}\left(\hat{q}^{\prime}\right) \phi^{\prime \prime} \Psi_{V}^{--}(\hat{q}), \not P \bar{\psi}_{P}^{--}\left(\hat{q}^{\prime}\right) \phi^{\prime \prime} \Psi_{V}^{++}(\hat{q})$, and $\not p \bar{\psi}_{P}^{--}\left(\hat{q}^{\prime}\right) \phi^{\prime \prime} \Psi_{V}^{--}(\hat{q})$ in the calculation of a transition amplitude $M_{f i}$ for $V \rightarrow P \gamma$ is done by using Eqs. (A3) and (A4) as

$$
\begin{aligned}
& \not P \bar{\psi}_{P}^{++}\left(\hat{q}^{\prime}\right) \phi^{\prime \prime} \Psi_{V}^{++}(\hat{q})=\frac{-i N_{P} N_{V}}{16 \omega_{1} \omega_{2} \omega_{1}^{\prime} \omega_{2}^{\prime}} \frac{M^{\prime}}{M} \phi_{P}\left(\hat{q}^{\prime}\right) \phi_{V}(\hat{q})\left[-i M\left(\omega_{1}^{\prime} \omega_{2}^{\prime}+m_{1} m_{2}+\hat{q}^{\prime 2}\right)\left(\omega_{1} m_{2}+m_{1} \omega_{2}\right) \not P \phi^{\prime \prime} \phi \not P \gamma_{5}\right. \\
& +i M^{2}\left(\omega_{1}^{\prime} \omega_{2}^{\prime}+m_{1} m_{2}+\hat{q}^{\prime 2}\right)\left(m_{1} \not P \phi^{\prime} \dot{\phi} \hat{\phi} \gamma_{5}+m_{2} \not \phi^{\prime \prime} \hat{\phi} \phi \gamma_{5}\right) \\
& -i M\left(\omega_{1}^{\prime} m_{2}+m_{1} \omega_{2}^{\prime}\right)\left(\omega_{1} \omega_{2}+m_{1} m_{2}\right) \not P \not P \phi^{\prime \prime} \phi \gamma_{5}+i M^{3}\left(\omega_{1}^{\prime} m_{2}+m_{1} \omega_{2}^{\prime}\right) \phi^{\prime \prime} \hat{\phi} \phi \hat{\phi} \phi \gamma_{5} \\
& -i M^{2}\left(\omega_{1}^{\prime} m_{2}+m_{1} \omega_{2}^{\prime}\right)\left(\omega_{1} \phi^{\prime \prime} \phi \vec{P} \hat{\phi} \gamma_{5}-\omega_{2} \phi^{\prime \prime} \hat{\phi} P \dot{\phi} \phi \gamma_{5}\right)-i M^{2}\left(m_{1}-m_{2}\right)\left(\omega_{1} \omega_{2}+m_{1} m_{2}\right) \not \hat{\phi}^{\prime} \phi^{\prime \prime} \phi \gamma_{5}
\end{aligned}
$$

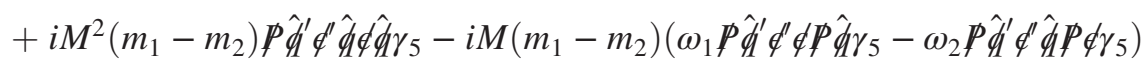

$$
\begin{aligned}
& -i M^{2}\left(\omega_{1} m_{2}+m_{1} \omega_{2}\right)\left(\omega_{1}^{\prime}+\omega_{2}^{\prime}\right) \hat{q}^{\prime} \phi^{\prime \prime} \phi P \gamma_{5}+i M^{3}\left(m_{1}\left(\omega_{1}^{\prime}+\omega_{2}^{\prime}\right) \hat{\phi}^{\prime} \phi^{\prime} \phi \hat{\phi} \gamma_{5}+m_{2}\left(\omega_{1}^{\prime}+\omega_{2}^{\prime}\right) \hat{\phi}^{\prime} \phi^{\prime} \hat{\phi} \phi \gamma_{5}\right] \text {, }
\end{aligned}
$$

$$
\begin{aligned}
& \not P \bar{\psi}_{P}^{--}\left(\hat{q}^{\prime}\right) \phi^{\prime} \Psi_{V}^{--}(\hat{q})=\frac{-i N_{P} N_{V}}{16 \omega_{1} \omega_{2} \omega_{1}^{\prime} \omega_{2}^{\prime}} \frac{M^{\prime}}{M} \phi_{P}\left(\hat{q}^{\prime}\right) \phi_{V}(\hat{q})\left[i M\left(\omega_{1}^{\prime} \omega_{2}^{\prime}+m_{1} m_{2}+\hat{q}^{\prime 2}\right)\left(\omega_{1} m_{2}+m_{1} \omega_{2}\right) \not P \phi^{\prime \prime} \phi \not P \gamma_{5}\right. \\
& +i M^{2}\left(\omega_{1}^{\prime} \omega_{2}^{\prime}+m_{1} m_{2}+\hat{q}^{\prime 2}\right)\left(m_{1} \not \boldsymbol{P} \phi^{\prime \prime} \dot{\phi} \hat{\phi} \gamma_{5}+m_{2} \not \phi^{\prime \prime} \hat{\phi} \phi \gamma_{5}\right) \\
& +i M\left(\omega_{1}^{\prime} m_{2}+m_{1} \omega_{2}^{\prime}\right)\left(\omega_{1} \omega_{2}+m_{1} m_{2}\right) \not P \not P \phi^{\prime \prime} \phi \gamma_{5}-i M^{3}\left(\omega_{1}^{\prime} m_{2}+m_{1} \omega_{2}^{\prime}\right) \phi^{\prime \prime} \hat{\phi} \phi \hat{\phi} \phi \gamma_{5} \\
& -i M^{2}\left(\omega_{1}^{\prime} m_{2}+m_{1} \omega_{2}^{\prime}\right)\left(\omega_{1} \phi^{\prime \prime} \phi \not \hat{\phi} \hat{\phi} \gamma_{5}-\omega_{2} \phi^{\prime \prime} \hat{q} \not \mathbf{\phi} \phi \gamma_{5}\right)-i M^{2}\left(m_{1}-m_{2}\right)\left(\omega_{1} \omega_{2}+m_{1} m_{2}\right) \not \hat{\phi}^{\prime} \phi^{\prime \prime} \phi \gamma_{5}
\end{aligned}
$$

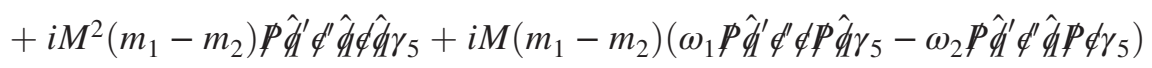

$$
\begin{aligned}
& -i M^{2}\left(\omega_{1} m_{2}+m_{1} \omega_{2}\right)\left(\omega_{1}^{\prime}+\omega_{2}^{\prime}\right) \hat{q}^{\prime} \phi^{\prime \prime} \phi P \gamma_{5}+i M^{3}\left(m_{1}\left(\omega_{1}^{\prime}+\omega_{2}^{\prime}\right) \hat{\phi}^{\prime} \phi^{\prime} \phi \hat{\phi} \gamma_{5}+m_{2}\left(\omega_{1}^{\prime}+\omega_{2}^{\prime}\right) \hat{q}^{\prime} \phi^{\prime \prime} \hat{\phi} \phi \gamma_{5}\right] \text {, }
\end{aligned}
$$

$$
\begin{aligned}
& \not p \bar{\psi}_{P}^{++}\left(\hat{q}^{\prime}\right) \phi^{\prime \prime} \Psi_{V}^{--}(\hat{q})=\frac{-i N_{P} N_{V}}{16 \omega_{1} \omega_{2} \omega_{1}^{\prime} \omega_{2}^{\prime}} \frac{M^{\prime}}{M} \phi_{P}\left(\hat{q}^{\prime}\right) \phi_{V}(\hat{q})\left[-i M\left(\omega_{1}^{\prime} \omega_{2}^{\prime}+m_{1} m_{2}+\hat{q}^{\prime 2}\right)\left(\omega_{1} m_{2}+m_{1} \omega_{2}\right) \not P \phi^{\prime \prime} \phi \not P \gamma_{5}\right. \\
& -i M^{2}\left(\omega_{1}^{\prime} \omega_{2}^{\prime}+m_{1} m_{2}+\hat{q}^{2}\right)\left(m_{1} \not \boldsymbol{P} \phi^{\prime \prime} \dot{\phi} \hat{\phi} \gamma_{5}+m_{2} \not \mathbf{P} \phi^{\prime} \hat{\phi} \phi \gamma_{5}\right) \\
& +i M\left(\omega_{1}^{\prime} m_{2}+m_{1} \omega_{2}^{\prime}\right)\left(\omega_{1} \omega_{2}+m_{1} m_{2}\right) P P \phi^{\prime \prime} \phi \gamma_{5}+i M^{3}\left(\omega_{1}^{\prime} m_{2}+m_{1} \omega_{2}^{\prime}\right) \phi^{\prime \prime} \hat{\phi} \phi \hat{\phi} \gamma \gamma_{5} \\
& +i M^{2}\left(\omega_{1}^{\prime} m_{2}+m_{1} \omega_{2}^{\prime}\right)\left(\omega_{1} \phi^{\prime} \phi \not P \hat{\phi} \gamma_{5}-\omega_{2} \phi^{\prime} \hat{\phi} \not \boldsymbol{P} \phi \gamma_{5}\right)-i M^{2}\left(m_{1}-m_{2}\right)\left(\omega_{1} \omega_{2}+m_{1} m_{2}\right) \not \hat{\phi}^{\prime} \phi^{\prime \prime} \phi \gamma_{5} \\
& +i M^{2}\left(m_{1}-m_{2}\right) \boldsymbol{P} \hat{q}^{\prime} \phi^{\prime \prime} \hat{\phi} \dot{\phi} \hat{\phi} \gamma_{5}+i M\left(m_{1}-m_{2}\right)\left(\omega_{1} \not \hat{\phi}^{\prime} \phi^{\prime \prime} \phi \vec{P} \hat{\phi} \gamma_{5}-\omega_{2} \not \hat{q}^{\prime} \phi^{\prime \prime} \hat{\phi} \not \boldsymbol{\phi} \phi \gamma_{5}\right) \\
& +i M^{2}\left(\omega_{1} m_{2}+m_{1} \omega_{2}\right)\left(\omega_{1}^{\prime}+\omega_{2}^{\prime}\right) \hat{\phi}^{\prime} \phi^{\prime \prime} \phi P \gamma_{5}+i M^{3}\left(m_{1}\left(\omega_{1}^{\prime}+\omega_{2}^{\prime}\right) \hat{\phi}^{\prime} \phi^{\prime \prime} \phi \hat{\phi} \gamma_{5}+m_{2}\left(\omega_{1}^{\prime}+\omega_{2}^{\prime}\right) \hat{\phi}^{\prime} \phi^{\prime \prime} \hat{\phi} \phi \gamma_{5}\right] \text {, }
\end{aligned}
$$


and

$$
\begin{aligned}
& \not p \bar{\psi}_{P}^{--}\left(\hat{q}^{\prime}\right) \phi^{\prime \prime} \Psi_{V}^{++}(\hat{q})=\frac{-i N_{P} N_{V}}{16 \omega_{1} \omega_{2} \omega_{1}^{\prime} \omega_{2}^{\prime}} \frac{M^{\prime}}{M} \phi_{P}\left(\hat{q}^{\prime}\right) \phi_{V}(\hat{q})\left[-i M\left(\omega_{1}^{\prime} \omega_{2}^{\prime}+m_{1} m_{2}+\hat{q}^{\prime 2}\right)\left(\omega_{1} m_{2}+m_{1} \omega_{2}\right) \not P \phi^{\prime \prime} \phi P \gamma_{5}\right. \\
& +i M^{2}\left(\omega_{1}^{\prime} \omega_{2}^{\prime}+m_{1} m_{2}+\hat{q}^{\prime 2}\right)\left(m_{1} \not P \phi^{\prime \prime} \phi \hat{\phi} \gamma_{5}+m_{2} \not P \phi^{\prime \prime} \hat{\phi} \phi \gamma_{5}\right) \\
& -i M\left(\omega_{1}^{\prime} m_{2}+m_{1} \omega_{2}^{\prime}\right)\left(\omega_{1} \omega_{2}+m_{1} m_{2}\right) \not P P \phi^{\prime} \phi \gamma_{5}-i M^{3}\left(\omega_{1}^{\prime} m_{2}+m_{1} \omega_{2}^{\prime}\right) \phi^{\prime \prime} \hat{\phi} \phi \hat{\phi} \gamma_{5} \\
& +i M^{2}\left(\omega_{1}^{\prime} m_{2}+m_{1} \omega_{2}^{\prime}\right)\left(\omega_{1} \phi^{\prime} \phi \not \vec{\phi} \hat{\phi} \gamma_{5}-\omega_{2} \phi^{\prime} \hat{\phi} \not \boldsymbol{P} \phi \gamma_{5}\right)-i M^{2}\left(m_{1}-m_{2}\right)\left(\omega_{1} \omega_{2}+m_{1} m_{2}\right) \not \hat{\phi}^{\prime} \phi^{\prime \prime} \phi \gamma_{5} \\
& +i M^{2}\left(m_{1}-m_{2}\right) \not \hat{P}^{\prime} \phi^{\prime} \hat{\phi} \dot{\phi} \hat{\phi} \gamma_{5}-i M\left(m_{1}-m_{2}\right)\left(\omega_{1} \not \hat{P}^{\prime} \phi^{\prime \prime} \phi \dot{P} \hat{\phi} \gamma_{5}-\omega_{2} \not \hat{\phi}^{\prime} \phi^{\prime} \hat{\phi} \not \boldsymbol{P} \phi \gamma_{5}\right) \\
& +i M^{2}\left(\omega_{1} m_{2}+m_{1} \omega_{2}\right)\left(\omega_{1}^{\prime}+\omega_{2}^{\prime}\right) \hat{\phi}^{\prime} \phi^{\prime \prime} \phi P \gamma_{5}+i M^{3}\left(m_{1}\left(\omega_{1}^{\prime}+\omega_{2}^{\prime}\right) \hat{\phi}^{\prime} \phi^{\prime} \phi \hat{\phi} \gamma_{5}+m_{2}\left(\omega_{1}^{\prime}+\omega_{2}^{\prime}\right) \hat{\phi}^{\prime} \phi^{\prime} \hat{\phi} \phi \gamma_{5}\right] .
\end{aligned}
$$

\section{Radiative decays through $V \rightarrow S \gamma$}

The ++ and -- components of a scalar meson wave function in Eq. (49) can be obtained through Eq. (38) as

$$
\begin{aligned}
& \psi_{S}^{++}\left(\hat{q}^{\prime}\right)=\frac{-N_{S}}{4 \omega_{1}^{\prime} \omega_{2}^{\prime}} \phi_{S}\left(\hat{q}^{\prime}\right)\left[-M\left(\omega_{1}^{\prime} \omega_{2}^{\prime}-m_{1} m_{2}+\hat{q}^{2}\right)-i\left(\omega_{1}^{\prime} m_{2}-m_{1} \omega_{2}^{\prime}\right) \not p-\left(\omega_{1}^{\prime} \not \hat{q}^{\prime}-\omega_{2}^{\prime} \hat{q}^{\prime} \not{P}\right)-i M\left(m_{1}+m_{2}\right) \hat{q}^{\prime},\right. \\
& \psi_{S}^{--}\left(\hat{q}^{\prime}\right)=\frac{-N_{S}}{4 \omega_{1}^{\prime} \omega_{2}^{\prime}} \phi_{S}\left(\hat{q}^{\prime}\right)\left[-M\left(\omega_{1}^{\prime} \omega_{2}^{\prime}-m_{1} m_{2}+\hat{q}^{2}\right)+i\left(\omega_{1}^{\prime} m_{2}-m_{1} \omega_{2}^{\prime}\right) \not P+\left(\omega_{1}^{\prime} \not \hat{q}^{\prime}-\omega_{2}^{\prime} \hat{q}^{\prime} \not \mathbf{P}\right)-i M\left(m_{1}+m_{2}\right) \hat{q}^{\prime} .\right.
\end{aligned}
$$

The corresponding adjoint wave functions are obtained by evaluating $\bar{\psi}_{S}^{ \pm \pm}\left(\hat{q}^{\prime}\right)=\gamma_{4}\left(\psi_{S}^{ \pm \pm}\left(\hat{q}^{\prime}\right)\right)^{+} \gamma_{4}$ as

$$
\begin{aligned}
& \bar{\psi}_{S}^{++}\left(\hat{q}^{\prime}\right)=\frac{-N_{S}}{4 \omega_{1}^{\prime} \omega_{2}^{\prime}} \phi_{S}\left(\hat{q}^{\prime}\right)\left[-M\left(\omega_{1}^{\prime} \omega_{2}^{\prime}-m_{1} m_{2}+\hat{q}^{\prime 2}\right)-i\left(\omega_{1}^{\prime} m_{2}-m_{1} \omega_{2}^{\prime}\right) \not p-\left(\omega_{1}^{\prime} \hat{q}^{\prime} \not \mathbf{P}-\omega_{2}^{\prime} \not \hat{q}^{\prime}\right)-i M\left(m_{1}+m_{2}\right) \hat{q}^{\prime},\right. \\
& \bar{\psi}_{S}^{--}\left(\hat{q}^{\prime}\right)=\frac{-N_{S}}{4 \omega_{1}^{\prime} \omega_{2}^{\prime}} \phi_{S}\left(\hat{q}^{\prime}\right)\left[-M\left(\omega_{1}^{\prime} \omega_{2}^{\prime}-m_{1} m_{2}+\hat{q}^{\prime 2}\right)+i\left(\omega_{1}^{\prime} m_{2}-m_{1} \omega_{2}^{\prime}\right) \not P+\left(\omega_{1}^{\prime} \hat{q}^{\prime} \not \boldsymbol{P}-\omega_{2}^{\prime} \not \hat{q}^{\prime}\right)-i M\left(m_{1}+m_{2}\right) \hat{q}^{\prime} .\right.
\end{aligned}
$$

The individual terms $\not p \bar{\psi}_{S}^{++}\left(\hat{q}^{\prime}\right) \phi^{\prime \prime} \Psi_{V}^{++}(\hat{q}), \not p \bar{\psi}_{S}^{++}\left(\hat{q}^{\prime}\right) \phi^{\prime \prime} \Psi_{V}^{--}(\hat{q}), \not P \bar{\psi}_{S}^{--}\left(\hat{q}^{\prime}\right) \phi^{\prime \prime} \Psi_{V}^{++}(\hat{q})$, and $\not P \bar{\psi}_{S}^{--}\left(\hat{q}^{\prime}\right) \phi^{\prime \prime} \Psi_{V}^{--}(\hat{q})$ in the transition amplitude $M_{f i}$ in Eq. (31) can be obtained as follows:

$$
\begin{aligned}
& \not p \bar{\psi}_{S}^{++}\left(\hat{q}^{\prime}\right) \phi^{\lambda^{\prime}} \psi_{V}^{++}(\hat{q})=\frac{-i N_{S} N_{V}}{16 \omega_{1} \omega_{2} \omega_{1}^{\prime} \omega_{2}^{\prime}} \phi_{S}\left(\hat{q}^{\prime}\right) \phi_{V}(\hat{q})\left[i M^{3}\left(\omega_{1}^{\prime} \omega_{2}^{\prime}-m_{1} m_{2}+\hat{q}^{\prime 2}\right)\left(\omega_{1} m_{2}+m_{1} \omega_{2}\right) \phi^{\lambda^{\prime}} \phi^{\lambda}\right. \\
& +i M^{2}\left(\omega_{1}^{\prime} \omega_{2}^{\prime}-m_{1} m_{2}+\hat{q}^{\prime 2}\right)\left(m_{1} \not \boldsymbol{P} \phi^{\lambda^{\prime}} \phi^{\lambda} \hat{\phi}+m_{2} \not \boldsymbol{P} \phi^{\lambda^{\prime}} \hat{\phi} \phi^{\lambda}\right) \\
& +i M^{3}\left(\omega_{1}^{\prime} m_{2}-m_{1} \omega_{2}^{\prime}\right)\left(\omega_{1} \omega_{2}+m_{1} m_{2}\right) \phi^{\lambda^{\prime}} \phi^{\lambda}-i M^{3}\left(\omega_{1}^{\prime} m_{2}-m_{1} \omega_{2}^{\prime}\right) \phi^{\lambda^{\prime}} \hat{\phi} \epsilon^{\lambda} \hat{\phi} \\
& +i M^{2}\left(\omega_{1}^{\prime} m_{2}-m_{1} \omega_{2}^{\prime}\right)\left(\omega_{1} \phi^{\lambda^{\prime}} \phi^{\lambda} \not \vec{P} \hat{q}-\omega_{2} \phi^{\lambda^{\prime}} \hat{\phi} \not P \phi^{\lambda}\right)-i M^{2}\left(\omega_{1} m_{2}+m_{1} \omega_{2}\right)\left(\omega_{1}^{\prime} \hat{q}^{\prime} \phi^{\lambda^{\prime}} \phi^{\lambda} \not P+\omega_{2}^{\prime} \hat{\phi}^{\prime} \phi^{\lambda^{\prime}} \phi^{\lambda} \not P\right) \\
& +i M^{3}\left(m_{1}\left(\omega_{1}^{\prime}+\omega_{2}^{\prime}\right) \hat{\phi}^{\prime} \phi^{\lambda^{\prime}} \phi^{\lambda} \hat{\phi}+m_{2}\left(\omega_{1}^{\prime}+\omega_{2}^{\prime}\right) \hat{\phi}^{\prime} \phi^{\lambda^{\prime}} \hat{\phi} \phi^{\lambda}\right)-i M^{2}\left(m_{1}+m_{2}\right)\left(\omega_{1} \omega_{2}+m_{1} m_{2}\right) \not \hat{\phi}^{\prime} \phi^{\lambda^{\prime}} \phi^{\lambda} \\
& \left.+i M^{2}\left(m_{1}+m_{2}\right) \not \hat{\phi}^{\prime} \phi^{\lambda^{\prime}} \hat{\phi} \phi^{\lambda} \hat{\phi}-i M^{3}\left(m_{1}+m_{2}\right)\left(\omega_{1} \hat{\phi}^{\prime} \phi^{\lambda^{\prime}} \phi^{\lambda} \hat{\phi}-\omega_{2} \hat{\phi}^{\prime} \phi^{\lambda^{\prime}} \hat{\phi} \phi^{\lambda}\right)\right] \text {, } \\
& +i M^{2}\left(\omega_{1}^{\prime} \omega_{2}^{\prime}-m_{1} m_{2}+\hat{q}^{\prime 2}\right)\left(m_{1} \not \boldsymbol{p} \phi^{\lambda^{\prime}} \phi^{\lambda} \hat{\phi}+m_{2} \not \phi^{\lambda^{\prime}} \hat{\phi} \phi^{\lambda}\right) \\
& -i M^{3}\left(\omega_{1}^{\prime} m_{2}-m_{1} \omega_{2}^{\prime}\right)\left(\omega_{1} \omega_{2}+m_{1} m_{2}\right) \phi^{\lambda^{\prime}} \phi^{\lambda}+i M^{3}\left(\omega_{1}^{\prime} m_{2}-m_{1} \omega_{2}^{\prime}\right) \phi^{\lambda^{\prime}} \hat{\phi} \epsilon^{\lambda} \hat{\phi} \\
& +i M^{2}\left(\omega_{1}^{\prime} m_{2}-m_{1} \omega_{2}^{\prime}\right)\left(\omega_{1} \phi^{\lambda^{\prime}} \phi^{\lambda} \not \vec{\phi} \hat{q}-\omega_{2} \phi^{\lambda^{\prime}} \hat{\phi} P \phi^{\lambda^{\lambda}}\right)-i M^{2}\left(\omega_{1} m_{2}+m_{1} \omega_{2}\right)\left(\omega_{1}^{\prime} \hat{q}^{\prime} \phi^{\lambda^{\prime}} \phi^{\lambda} \not \mathbf{P}+\omega_{2}^{\prime} \hat{q}^{\prime} \phi^{\lambda^{\prime}} \phi^{\lambda} \not \mathbf{P}\right) \\
& -i M^{3}\left(m_{1}\left(\omega_{1}^{\prime}+\omega_{2}^{\prime}\right) \hat{q}^{\prime} \phi^{\lambda^{\prime}} \phi^{\lambda} \hat{\phi}+m_{2}\left(\omega_{1}^{\prime}+\omega_{2}^{\prime}\right) \hat{q}^{\prime} \phi^{\lambda^{\prime}} \hat{\phi} \phi^{\lambda}\right)-i M^{2}\left(m_{1}+m_{2}\right)\left(\omega_{1} \omega_{2}+m_{1} m_{2}\right) \dot{P}^{\prime} \hat{\phi}^{\prime} \phi^{\lambda^{\prime}} \phi^{\lambda} \\
& \left.+i M^{2}\left(m_{1}+m_{2}\right) \not \hat{\phi}^{\prime} \phi^{\phi^{\prime}} \hat{\phi} \phi^{\lambda} \hat{\phi}+i M^{3}\left(m_{1}+m_{2}\right)\left(\omega_{1} \hat{\phi}^{\prime} \phi^{\lambda^{\prime}} \phi^{\lambda} \hat{\phi}-\omega_{2} \hat{\phi}^{\prime} \phi^{\phi^{\prime}} \hat{\phi} \phi^{\lambda}\right)\right] \text {, }
\end{aligned}
$$




$$
\begin{aligned}
& \not P \bar{\psi}_{S}^{++}\left(\hat{q}^{\prime}\right) \phi^{\lambda^{\prime}} \psi_{V}^{--}(\hat{q})=\frac{-i N_{S} N_{V}}{16 \omega_{1} \omega_{2} \omega_{1}^{\prime} \omega_{2}^{\prime}} \phi_{S}\left(\hat{q}^{\prime}\right) \phi_{V}(\hat{q})\left[-i M^{3}\left(\omega_{1}^{\prime} \omega_{2}^{\prime}-m_{1} m_{2}+\hat{q}^{\prime 2}\right)\left(\omega_{1} m_{2}+m_{1} \omega_{2}\right) \phi^{\lambda^{\prime}} \phi^{\lambda \lambda}\right. \\
& +i M^{2}\left(\omega_{1}^{\prime} \omega_{2}^{\prime}-m_{1} m_{2}+\hat{q}^{\prime 2}\right)\left(m_{1} \not P \phi^{\lambda^{\prime}} \phi^{\lambda} \hat{\phi}+m_{2} \not P \phi^{\lambda^{\prime}} \hat{\phi} \phi \phi^{\lambda}\right) \\
& +i M^{3}\left(\omega_{1}^{\prime} m_{2}-m_{1} \omega_{2}^{\prime}\right)\left(\omega_{1} \omega_{2}+m_{1} m_{2}\right) \phi^{\lambda^{\prime}} \phi^{\lambda}-i M^{3}\left(\omega_{1}^{\prime} m_{2}-m_{1} \omega_{2}^{\prime}\right) \phi^{\lambda^{\prime}} \hat{\phi} \epsilon^{\lambda} \hat{\phi} \\
& -i M^{2}\left(\omega_{1}^{\prime} m_{2}-m_{1} \omega_{2}^{\prime}\right)\left(\omega_{1} \phi^{\lambda^{\prime}} \phi^{\lambda} \not \mathbf{P} \hat{\phi}-\omega_{2} \phi^{\lambda^{\prime}} \hat{\phi} \not P \phi^{\lambda}\right)+i M^{2}\left(\omega_{1} m_{2}+m_{1} \omega_{2}\right)\left(\omega_{1}^{\prime} \hat{\phi}^{\prime} \phi^{\lambda^{\prime}} \phi^{\lambda} \not P+\omega_{2}^{\prime} \hat{\phi}^{\prime} \phi^{\lambda^{\prime}} \phi^{\lambda} \not P\right) \\
& +i M^{3}\left(m_{1}\left(\omega_{1}^{\prime}+\omega_{2}^{\prime}\right) \hat{\phi}^{\prime} \phi^{\lambda^{\prime}} \phi^{\lambda} \hat{\phi}+m_{2}\left(\omega_{1}^{\prime}+\omega_{2}^{\prime}\right) \hat{\phi}^{\prime} \phi^{\lambda^{\prime}} \hat{\phi} \phi^{\lambda}\right)-i M^{2}\left(m_{1}+m_{2}\right)\left(\omega_{1} \omega_{2}+m_{1} m_{2}\right) \not \hat{\phi}^{\prime} \phi^{\lambda^{\prime}} \phi^{\lambda} \\
& \left.+i M^{2}\left(m_{1}+m_{2}\right) \not \hat{\phi}^{\prime} \phi^{\lambda^{\prime}} \hat{\phi} \phi^{\lambda} \hat{\phi}+i M^{3}\left(m_{1}+m_{2}\right)\left(\omega_{1} \hat{\phi}^{\prime} \phi^{\lambda^{\prime}} \phi^{\lambda} \hat{\phi}-\omega_{2} \hat{\phi}^{\prime} \phi^{\lambda^{\prime}} \hat{\phi} \phi^{\lambda \lambda}\right)\right] \text {, }
\end{aligned}
$$

and

$$
\begin{aligned}
& \not P \bar{\psi}_{S}^{--}\left(\hat{q}^{\prime}\right) \phi^{\lambda^{\prime}} \psi_{V}^{++}(\hat{q})=\frac{-i N_{S} N_{V}}{16 \omega_{1} \omega_{2} \omega_{1}^{\prime} \omega_{2}^{\prime}} \phi_{S}\left(\hat{q}^{\prime}\right) \phi_{V}(\hat{q})\left[i M^{3}\left(\omega_{1}^{\prime} \omega_{2}^{\prime}-m_{1} m_{2}+\hat{q}^{\prime 2}\right)\left(\omega_{1} m_{2}+m_{1} \omega_{2}\right) \phi^{\lambda^{\prime}} \phi^{\lambda \lambda}\right. \\
& +i M^{2}\left(\omega_{1}^{\prime} \omega_{2}^{\prime}-m_{1} m_{2}+\hat{q}^{\prime 2}\right)\left(m_{1} \not \phi^{\lambda^{\prime}} \phi^{\lambda} \hat{\phi}+m_{2} \not \phi^{\lambda^{\prime}} \hat{\phi} \phi^{\lambda}\right) \\
& -i M^{3}\left(\omega_{1}^{\prime} m_{2}-m_{1} \omega_{2}^{\prime}\right)\left(\omega_{1} \omega_{2}+m_{1} m_{2}\right) \phi^{\lambda^{\prime}} \phi^{\lambda}+i M^{3}\left(\omega_{1}^{\prime} m_{2}-m_{1} \omega_{2}^{\prime}\right) \phi^{\lambda^{\prime}} \hat{\phi} \epsilon^{\lambda} \hat{\phi} \\
& -i M^{2}\left(\omega_{1}^{\prime} m_{2}-m_{1} \omega_{2}^{\prime}\right)\left(\omega_{1} \phi^{\lambda^{\prime}} \phi^{\lambda} \not \mathbf{P} \hat{\phi}-\omega_{2} \phi^{\lambda^{\prime}} \hat{\phi} \boldsymbol{P} \phi^{\lambda}\right)+i M^{2}\left(\omega_{1} m_{2}+m_{1} \omega_{2}\right)\left(\omega_{1}^{\prime} \hat{\phi}^{\prime} \phi^{\lambda^{\prime}} \phi^{\lambda} \not P+\omega_{2}^{\prime} \hat{\phi}^{\prime} \phi^{\lambda^{\prime}} \phi^{\lambda} \not P\right) \\
& -i M^{3}\left(m_{1}\left(\omega_{1}^{\prime}+\omega_{2}^{\prime}\right) \hat{\phi}^{\prime} \phi^{\phi^{\prime}} \phi^{\lambda} \hat{\phi}+m_{2}\left(\omega_{1}^{\prime}+\omega_{2}^{\prime}\right) \hat{\phi}^{\prime} \phi^{\lambda^{\prime}} \hat{\phi} \phi^{\lambda}\right)-i M^{2}\left(m_{1}+m_{2}\right)\left(\omega_{1} \omega_{2}+m_{1} m_{2}\right) \not \hat{\phi}^{\prime} \phi^{\lambda^{\prime}} \phi^{\lambda \lambda} \\
& \left.+i M^{2}\left(m_{1}+m_{2}\right) \not \hat{\phi}^{\prime} \phi^{\lambda^{\prime}} \hat{\phi} \phi^{\lambda} \hat{\phi}-i M^{3}\left(m_{1}+m_{2}\right)\left(\omega_{1} \hat{\phi}^{\prime} \phi^{\lambda^{\prime}} \phi^{\lambda} \hat{\phi}-\omega_{2} \hat{\phi}^{\prime} \phi^{\lambda^{\prime}} \hat{\phi} \phi^{\lambda}\right)\right] \text {. }
\end{aligned}
$$

[1] K. M. Ecklund et al. (CLEO Collaboration), Phys. Rev. D 78, 091501 (2008).

[2] B. Auger et al. (BABAR Collaboration), Phys. Rev. Lett. 103, 161801 (2009).

[3] K. F. Chen et al. (Belle Collaboration), Phys. Rev. D 82, 091106(R) (2010).

[4] K. W. Edwards et al. (CLEO Collaboration), Phys. Rev. Lett. 86, 30 (2001).

[5] K. A. Olive et al. (Particle Data Group), Chin. Phys. C 38, 090001 (2014).

[6] N. Brambila et al., arXiv:hep-ph/0412158.

[7] E. Eichten, S. Godfrey, H. Mahlke, and J. L. Rosner, Rev. Mod. Phys. 80, 1161 (2008).

[8] N. Brambila, Y. Jia, and A. Vairo, Phys. Rev. D 73, 054005 (2006).

[9] A. Pineda and J. Segovia, Phys. Rev. D 87, 074024 (2013).

[10] M. Li, Y. Li, P. Maris, and J. P. Vary, Phys. Rev. D 98, 034024 (2018).

[11] H. M. Choi, Phys. Rev. D 75, 073016 (2007).

[12] D. Becirevic and F. Sanfillipo, J. High Energy Phys. 01 (2013) 028.

[13] G. C. Donald, C. T. H. Davies, R. J. Dowdall, E. Follana, K. Hornbostel, J. Koponen, G. P. Lepage, and C. Mc Neile, Phys. Rev. D 86, 094501 (2012).

[14] A. N. Mitra and S. Bhatnagar, Int. J. Mod. Phys. A 07, 121 (1992).
[15] A. N. Mitra and B. M. Sodermark, Nucl. Phys. A695, 328 (2001).

[16] J. Carbonell and V. A. Karmanov, Phys. Rev. D 91, 076010 (2015).

[17] H. Negash and S. Bhatnagar, Intl. J. Mod. Phys. E 25, 1650059 (2016).

[18] S. Bhatnagar and L. Alemu, Phys. Rev. D 97, 034021 (2018).

[19] E. Gebrehana, S. Bhatnagar, and H. Negash, Phys. Rev. D 100, 054034 (2019).

[20] H. Negash and S. Bhatnagar, Adv. High Energy Phys. 2017, 7306825 (2017).

[21] V. A. Karmanov, J. Karbonell, and H. Sazdjian, EPJ Web Conf. 204, 01014 (2019).

[22] Z. K. Geng, T. Wang, Y. Jiang, G. Li, X.-Z. Tan, and G.-L. Wang, Phys. Rev. D 99, 013006 (2019).

[23] T. Fredrico, G. Salme, and M. Viviani, Phys. Rev. D 89, 016010 (2014).

[24] C. H. Chang, J. K. Chen, and G. L. Wang, Commun. Theor. Phys. (Beijing) 46, 467 (2006).

[25] J. He, Eur. Phys. J. C 79, 393 (2019).

[26] S. Bhatnagar, J. Mahecha, and Y. Mengesha, Phys. Rev. D 90, 014034 (2014).

[27] T. Wang, Y. Jiang, W. L. Ju, H. Yuan, and G. L. Wang, J. High Energy Phys. 03 (2016) 209.

[28] C. H. L. Smith, Ann. Phys. (N.Y.) 53, 521 (1969).

[29] R. Alkofer and L. V. Smekel, Phys. Rep. 353, 281 (2002). 
[30] S. Bhatnagar and S.-Y. Li, J. Phys. G 32, 949 (2006).

[31] S. Bhatnagar, S.-Y. Li, and J. Mahecha, Int. J. Mod. Phys. E 20, 1437 (2011).

[32] H. J. Munczek and P. Jain, Phys. Rev. D 46, 438 (1992); P. Jain and H. J. Munczek, Phys. Rev. D 48, 5403 (1993).

[33] V. Sauli, Phys. Rev. D 86, 096004 (2012).

[34] J. J. Dudek, R. G. Edwards, and D. G. Richards, Phys. Rev. D 73, 074507 (2006).

[35] K. Nakamura et al. (Particle Data Group), J. Phys. G 37, 075021 (2010).

[36] S. F. Radford and W. W. Repko, Phys. Rev. D 75, 074031 (2007).

[37] D. Ebert, R. N. Faustov, and V. O. Galkin, Phys. Rev. D 67, 014027 (2003).
[38] K. A. Olive et al. (Particle Data Group), Chin. Phys. C 38, 090001 (2014).

[39] S. S. Gershtein, V. V. Kiselev, A. K. Likhoded, and A. V. Tkabladze, Phys. Rev. D 51, 3613 (1995).

[40] M. Tanabashi et al. (Particle Data Group), Phys. Rev. D 98, 030001 (2018).

[41] M. Priyadarshini, P. C. Dash, S. Kar, S. Patra, and N. Barik, Phys. Rev. D 94, 113011 (2016).

[42] J. N. Pandya, N. R. Soni, N. Devlani, and A. K. Rai, Chin. Phys. C 39, 123101 (2015).

[43] L. P. Fulcher, Phys. Rev. D 60, 074006 (1999).

[44] C. Patrignani et al. (Particle Data Group), Chin. Phys. C 40, 100001 (2016). 